\title{
Inertial Effect on Interaction between Two Earthquake Faults
}

\author{
Jeen-Hwa Wang \\ Institute of Earth Sciences, Academia Sinica, Taipei, Taiwan \\ Article history: received February 21, 2019; accepted October 28, 2019
}

\begin{abstract}
This study is focused on the inertial effect on slip of and interaction between two earthquake faults based on a two-body slider-slider model in the presence of thermal-pressurized slip-weakening friction and viscosity. The ratio $\mu=m_{2} / m_{1}$, where $m_{2}$ and $m_{1}$ are the masses of sliders 1 and 2 , respectively, is the model parameter to represent the inertial effect. Simulation results show that $\mu$ is important on interaction between the two sliders. Foreshocks and aftershocks/afterslip can be generated on slider 1 or slider 2 when $\mu \neq 1$. Slider 2 behaves like a slow event when $\mu \geq 50$. Other model parameters are $s$ (the stiffness ratio), $\phi=f_{o 2} / f_{01}$ (where $f_{o i}$ is the normalized static friction force on the $i$-th slider, $i=1,2$ ), $\psi=U_{c 2} / U_{c 1}$ (where $U_{c i}$ is the normalized characteristic displacement of friction law on the $i$-th slider), and $\gamma=\eta_{2} / \eta_{\mathrm{i}}$ (where $\eta_{\mathrm{i}}$ is the normalized viscosity coefficient between the $i$-th slider and the background plate). The $\phi, \psi$, and $\gamma$ are the major factors and $s$ is minor one in causing time delay between the two sliders. Slider 2 cannot move when $\phi$ is higher than a critical value which depends on other model parameters. Interaction and the patterns of motions of the two sliders are different between $\psi<1$ and $\psi>1$. The presence of viscosity may increase the predominant period of a slider.
\end{abstract}

Keywords: Two-body spring-slider model, displacement, velocity, inertia, seismic coupling, thermalpressurized slip-weakening friction, viscosity.

\section{Introduction}

There is a significant problem of earthquake dynamics [e.g., King et al., 2001; Steacy et al., 2005; Wang et al., 2013]: "How do two faults interact each other during faulting?". The problem can be answered through field surveys, laboratory experiments, and theoretical modeling. It is lack of a comprehensive physical model to approach earthquake dynamics. Nevertheless, some models, including the crack model and spring-slider model, have been built up to reach the purpose. There are numerous factors of earthquake dynamics [see Bizzarri 2009; Wang 2016; and cited references therein]. Among these factors, friction and viscosity are usually regarded as two major ones. The inertial effect is rarely taken into account in the dislocation, crack, and statistical physics models. In order to include the inertial effect, Burridge and Knopoff [1967] proposed a 1-D spring-slider model to approach earthquake dynamics. Wang [2000, 2012] extended it to a 2-D version. The 1-D and 2-D models and their modified or simplified versions in the presence of friction and viscosity have been long and widely used for modelling earthquakes [Wang 2007, 2016, 2017a,b; and cited references therein]. But, the inertial effect has not been explicitly considered as an important factor in earthquake dynamics. 


\section{Jeen-Hwa Wang}

Since the used friction laws are nonlinear, the dynamical model itself may behave nonlinearly. A nonlinear dynamical system can show chaotic behavior under some conditions [Thompson and Stewart 1986; Turcotte 1992]. This indicates that the system is highly sensitive to initial conditions and thus a small difference in initial conditions, including those due to rounding errors in numerical calculations, produces unexpected outputs. This is known as (deterministic) chaos [Lorenz 1963].

The few-body models with rheology [e.g., Nur, 1978; Gu et al., 1984; Perez Pascual and Lomnitz-Adler, 1988] have long been applied to explore fault dynamics. A review of related studies made based on different springslider models can be seen in Wang [2017a]. Some studies based on the two-body model are given here: (1) the model with simple static/dynamic friction [e.g., Nussbaum and Ruina 1987; Huang and Turcotte 1990]; (2) the model with velocity-dependent friction [e.g., Huang and Turcotte 1992; de Sousa Vieira 1999; Galvanetto 2002]; (3) the model with rate- and state-dependent friction [e.g., Abe and Kato 2013]; (4) the model with velocityweakening friction [Brun and Gomez 1994]; (5) the model with slip-weakening friction [e.g., Wang 2017b].

In this work, I will investigate the inertial effect on interaction between two earthquake faults based on a two-body spring-slider model with thermal-pressurized slip-weakening friction and viscosity when the seismic coupling is weak. Of course, the effects due to seismic coupling, friction, and viscosity will also be discussed.

\section{Model}

\subsection{Two-body spring-slider model}

The description of this model can be found in Wang [2017b]. The model consists of two sliders of mass $m_{i}(i=1,2)$, a coil spring of strength $K$ linking two sliders, and two leaf springs of strength $L_{i}(i=1,2)$ pulling the respect sliders by a moving plate with a constant velocity $v_{P}$ (Figure 1). The moving plate provides the driving force, $L_{i} v P t$, on the sliders. At time $t=0$, the two sliders rest in an equilibrium state. The $i$-th slider is located at position $u_{i}$, measured with respect to its initial equilibrium position, along the $x$-axis. Each slider is subjected to a frictional force, $F_{i}\left(u_{i}, v_{i}\right)$, where $u_{i}$ and $v_{i}=d u_{i} / d t$ are the displacement and velocity of the $i$-th slider, respectively, and a velocity-dependent viscous force, $\phi\left(v_{i}\right)$. Hence, the equations of motion are

$$
\begin{aligned}
& m_{1}\left(d^{2} u_{1} / d t^{2}\right)=K\left(u_{2}-u_{1}\right)-L_{1}\left(u_{1}-v p t\right)-F_{1}\left(u_{1}, v_{1}\right)-\phi\left(v_{1}\right) \\
& m_{2}\left(d^{2} u_{2} / d t^{2}\right)=K\left(u_{1}-u_{2}\right)-L_{2}\left(u_{2}-v p t\right)-F_{2}\left(u_{2}, v_{2}\right)-\phi\left(v_{2}\right) .
\end{aligned}
$$

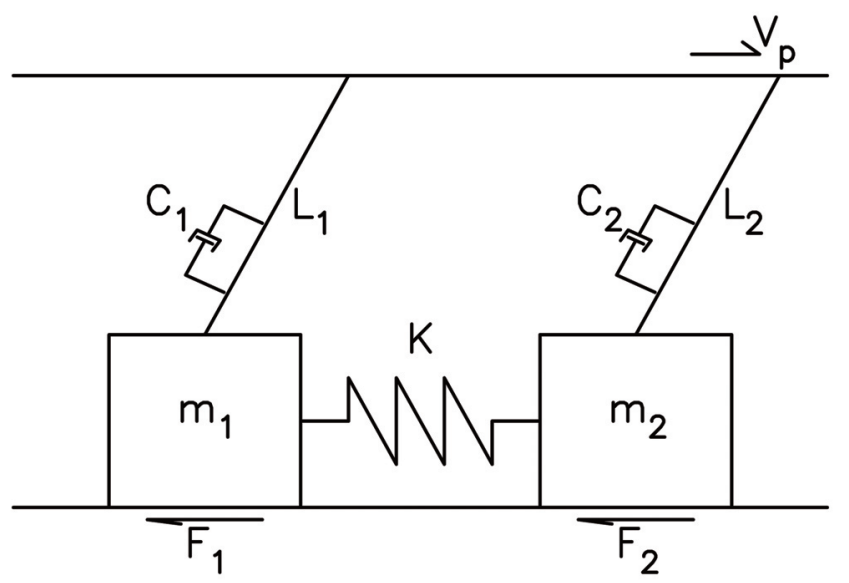

Figure 1. A two-body spring-slider model: $F_{i}=$ the friction force at the $i$-th slider $(i=1,2), m_{i}=$ the mass of the $i$-th slider, $K=$ the stiffness between two sliders, $L_{i}=$ the stiffness between the $i$-th slider and the moving plate, $C_{i}=$ the viscosity coefficient between the $i$-th slider and the moving plate, and $v_{p}=$ the velocity of the moving plate, and $u_{i}$ is the displacement of the $i$-th slider. 
The total forces in Equation (1) are null when the two sliders do not slide, and then the initial conditions are $u_{\mathrm{i}}=0$ and $v_{i}=0$. Considering the two sliders to represent two faults in a small area, the coupling between the moving plate and each slider should be equal, thus giving $L_{1}=L_{2}=L$. The ratio $s=K / L$ has been defined as the stiffness ratio of a spring-slider system by Wang [1995]. Wang [2007, 2017b] only considered $m_{1}=m_{2}$. This study will focus on the condition of $m_{1} \neq m_{2}$. Note that Equation (1) exhibits only the strike-slip component and, thus, cannot completely represent earthquake ruptures, which also consist of transpressive components. Nevertheless, this model can still help us to catch significant information of earthquake ruptures.

\subsection{Friction caused by thermal pressurization}

The friction force is produced from thermal pressurization proposed by Rice [2006]. He suggested two endmembers models for thermal pressurization: the adiabatic-undrained-deformation (AUD) model and slip-on-a-plane (SOP) model. Since the SOP model is based on a constant velocity, it will not be considered below. For the AUD model, the shear stress-slip function, $\tau(u)$, is $\tau(u)=f\left(\sigma_{n}-p_{o}\right) \exp \left(-u / u_{c}\right)$, thus exhibiting an exponential decay of $\tau(u)$ with $u$. The characteristic displacement is $u_{c}=\rho_{f} C_{v} h / \mu_{f} \Lambda$, where $\rho_{f}, C_{v}, h, \mu_{f}$, and $\Lambda$ are, respectively, the fluid density, heat capacity (in $J /{ }^{\circ} \mathrm{C} / \mathrm{kg}$ ), the thickness, frictional strength, and the undrained pressurization factor of the fault zone. Here, a slip-weakening friction law: $F(u)=F_{0} \exp \left(-u / u_{c}\right)$ based on the AUD model is used for modelling. Figure 2 displays the variations of $F(u)$ versus $u$ for five values of $u_{c}$, i.e., $0.1,0.3,0.5,0.7$, and $0.9 \mathrm{~m} . F(u)$ decreases with increasing $u$ and decreases faster for smaller $u_{c}$ than for larger $u_{c}$. The force drop for a fixed displacement decreases with increasing $u_{c}$.

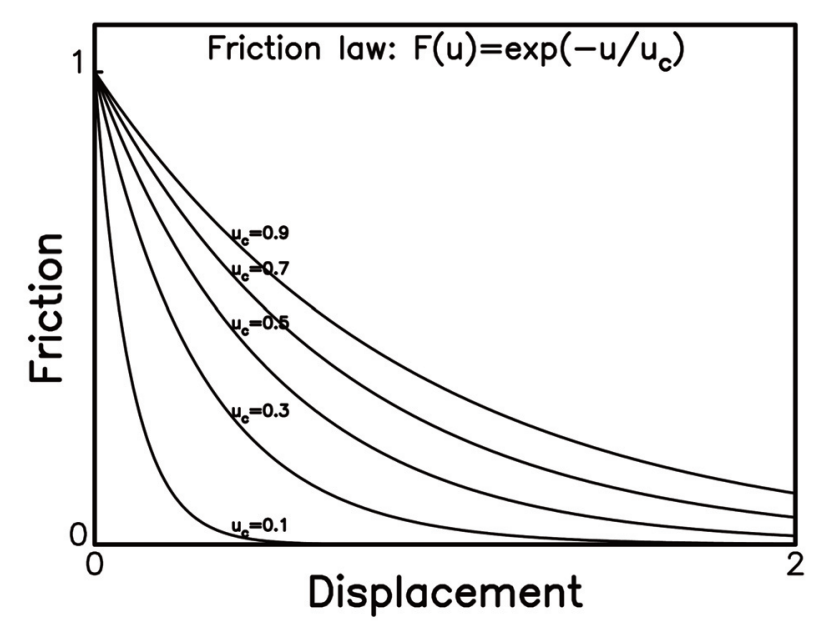

Figure 2. The variations of friction force with sliding displacement for $u_{c}=0.1,0.3,0.5,0.7$, and $0.9 \mathrm{~m}$.

\subsection{Viscosity}

Jeffreys [1942] first emphasized the importance of viscosity of fault-zone materials on earthquake dynamics. Related studies can be seen in some articles [e.g., Chen and Knopoff 1986; Wang 2007, 2016, 2017a,b]. Two simple models, i.e., the Kelvin-Voigt model and Maxwell model, are commonly used to describe the viscous materials [Jaeger and Cook 1977; Hudson 1980]. The former is more appropriate than the latter to be applied to the seismological problems [Hudson 1980; Brietzkel and Ben-Zion 2006; Wang 2016, 2017a,b], and thus the former is taken hereafter. In order to directly implement viscosity in a dynamical system, Wang [2016] proposed the damping coefficient $C$ of a sphere of radius $R$ in a fluid of viscosity $v$ to be $C=6 \pi R v$ based on Stokes" law [Kittel et al. 1968] and he used it to represent viscosity. Hence, the viscous force is given by $\phi=C v$, in which the unit of $C$ is $N /(m / s)$. The viscosity is shown by $C_{1}$ on slider 1 and $C_{2}$ on slider 2 in Figure 2. 


\section{Jeen-Hwa Wang}

\subsection{Inertial effect}

Inertia is the resistance of an object to any change in its velocity due to external forces. Since the mass, $m$, of an object denotes something like an amount of substance or quantity of matter, the mass is the quantitative measure of inertia of an object. In Equation (1), $m_{1}$ and $m_{2}$ denote the inertia of sliders 1 and 2 , respectively. In the followings, the ratio of $m_{2}$ to $m_{1}$, i.e., $\mu=m_{2} / m_{1}$, is taken to represent the inertial effect on interaction between the two sliders.

\section{Normalization of equation of motions}

Substituting the friction law and the viscous law into Equation (1) leads to

$$
\begin{aligned}
& m_{1}\left(d^{2} u_{1} / d t^{2}\right)=K\left(u_{2}-u_{1}\right)-L\left(u_{1}-v P t\right)-F_{O 1} \exp \left(-u_{1} / u_{C 1}\right)-C_{1} d u_{1} / d t \\
& m_{2}\left(d^{2} u_{2} / d t^{2}\right)=K\left(u_{1}-u_{2}\right)-L\left(u_{2}-v P t\right)-F_{O 2} \exp \left(-u_{2} / u_{C 2}\right)-C_{2} d u_{2} / d t
\end{aligned}
$$

To easily deal with the problem, Equation (2) is normalized based on the following normalization factors: $m_{1}=m$, $m_{2}=\mu m, F_{01}=F_{0}, F_{o 2}=\phi F_{0}, D_{0}=F_{0} / L, \omega_{o 1}=\omega_{0}=(L / m)^{1 / 2}, \omega_{0} 2=\mu^{-1 / 2} \omega_{0}, \tau=\omega_{0} t, u_{c 1}=u_{c}, u_{c 2}=\psi u_{c}, U_{c 1}=u c / D_{o}, U_{c 2}=\psi u_{0} / D_{o}$, $f_{o 1}=f_{o}=F_{0} / D_{o}, f_{o 2}=\phi f_{o}, \eta_{1}={ }_{c 1} \omega_{0} / L, \eta_{2}=C_{2} \mu^{-1 / 2} \omega_{0} / L, \gamma=\eta_{2} / \eta_{1}$, and $V_{P}=v_{P} / D_{0} \omega_{0}$. Defining $U_{i}=u_{i} / D_{o}$ and $V_{i}=d U_{i} / d \tau$ leads to $d u_{i} / d t=\left[F_{o} /(m L)^{1 / 2}\right] d U_{i} / d \tau$ and $d^{2} u_{i} / d t^{2}=\left(F_{o} / m\right) d^{2} U_{i} / d \tau^{2}$. Inserting these normalization parameters with $f_{\mathrm{o}}=1$ into Equation (2) results in:

$$
\begin{gathered}
d^{2} U_{1} / d \tau^{2}=s\left(U_{2}-U_{1}\right)-\left(U_{1}-V_{P} \tau\right)-\exp \left(-U_{1} / U_{c 1}\right)-\eta_{1} d U_{1} / d \tau \\
d^{2} U_{2} / d \tau^{2}=\left[s\left(U_{1}-U_{2}\right)-\left(U_{2}-V_{P} \tau\right)-\phi \exp \left(-U_{2} / U_{c 2}\right)-\eta_{2} d U_{2} / d \tau\right] / \mu .
\end{gathered}
$$

\section{Numerical simulations}

\section{1 four first-order dierential equations}

In order to numerically solve Equation (3), the equation is re-written to be a set of four first-order differential equations. Let $y_{1}=U_{1}, y_{2}=U_{2}, y_{3}=d U_{1} / d \tau$, and $y_{4}=d U_{2} / d \tau$. Equation (3) becomes:

$$
\begin{gathered}
d y_{1} / d \tau=y_{3} \\
d y_{2} / d \tau=y_{4} \\
d y_{3} / d \tau=-(s+1) y_{1}+s y_{2}-\exp \left(-y_{1} / U_{c 1}\right)-\eta_{1} y_{3}+V_{P} \tau \\
d y_{4} / d \tau=\left[s y_{1}-(s+1) y_{2}-\phi \exp \left(-y_{2} / \psi U_{c 1}\right)-\gamma \eta_{1} y_{4}+V_{P} \tau\right] / \mu .
\end{gathered}
$$

The solutions of Equation (4) exist within a five-dimensional domain controlled by five model parameters, i.e., $s, \mu, \gamma, \phi$, and $\psi$ when the values of $F_{o}, U_{c 1}$, and $\eta_{1}$ are fixed. Since it is difficult to analytically solve Equation (4), only numerical simulations using the fourth-order Runge-Kutta method [Press et al., 1986] will be performed in this study. Note that the sliders are restricted to move only along the positive direction, that is, $V_{\mathrm{i}} \geq 0$ and $U_{\mathrm{i}} \leq 0(i=1,2)$.

\subsection{Phase portrait}

The phase portrait is a plot of a physical quantity, for example $Y$, versus another, for example $X$, of an object in a dynamical system represented by $Y=f(X)$ [Thompson and Stewart 1986]. The intersection point of $f(X)$ and the 
bisection line, i.e., $Y=f(X)=X$, is called the fixed point, $X_{f}$. It is an attractor if the $f(X)$ is continuously differentiable in an open domain near it with $\left|f^{\prime}\left(X_{f}\right)\right|<1$. In other words, for any value of $X$ in the domain which is close enough to $X_{f}$, the iterated function sequences, i.e., $X, f(X), f^{2}(X), f^{3}(X), \ldots$, converges to $x_{f}$. Some attractors can yield chaos. In the following study, " $Y$ " represents $V / V_{\max }$ and " $X$ " denotes $U / U_{\max }$ for both sliders as shown in Figures 3-17.
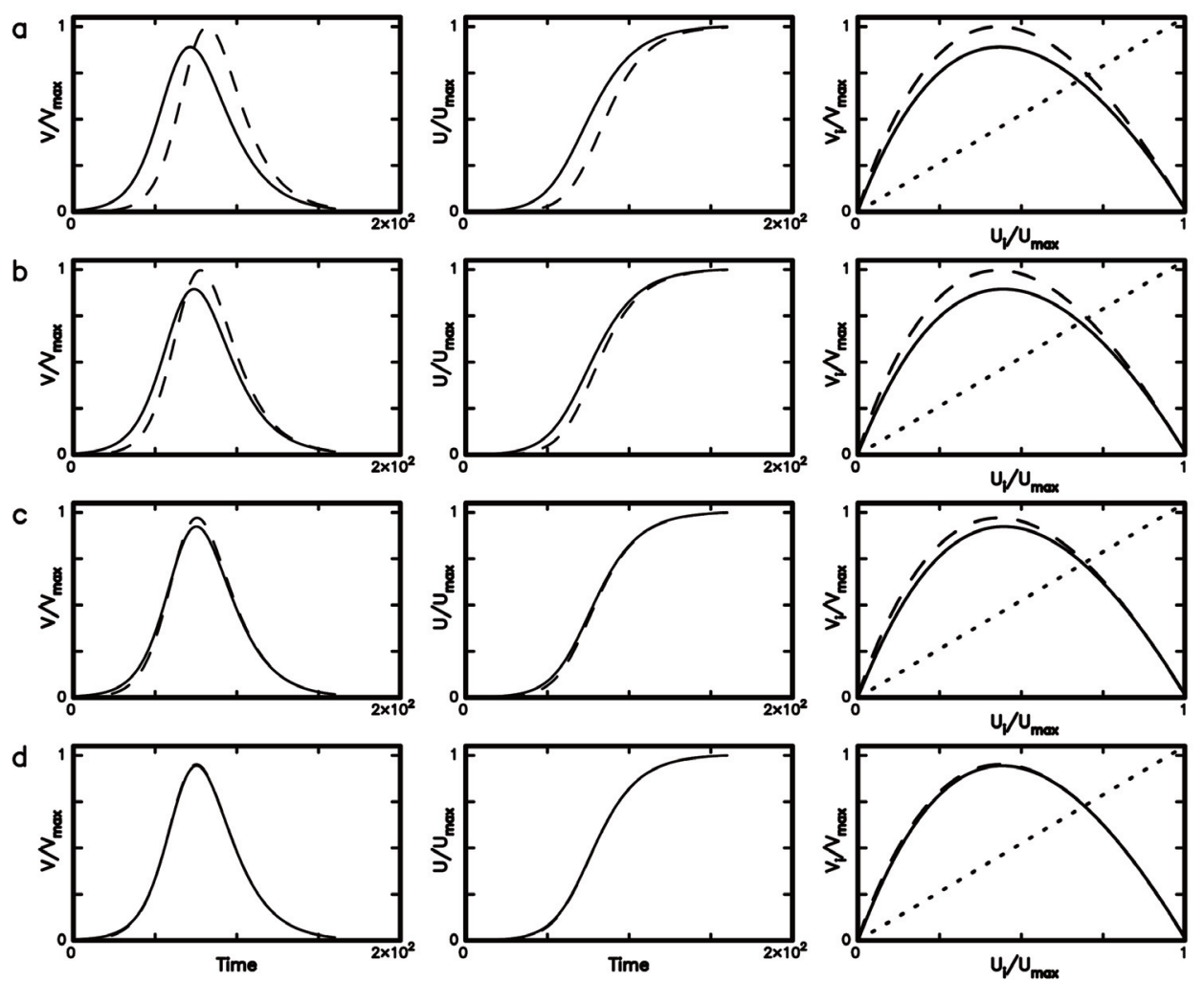

Figure 3. The time sequences of $V / V_{\max }$ and $U / U_{\max }$ and the phase portraits of $V / V_{\max }$ versus $U / U_{\max }$ of the two sliders (solid line for slider 1 and dashed line for slider 2): (a) for $s=0.06$, (b) for $s=0.12$, (c) for $s=0.30$, and (d) for $s=0.48$ when $\mu=1, f_{o 1}=1.0$ and $f_{o 2}=1.0$ (with $\phi=1$ ), $U_{c 1}=0.5$ and $U_{c 2}=0.5$ (with $\psi=1$ ), and $\eta_{1}=0$ and $\eta_{2}=0$ (with $\gamma=1$ ).

\subsection{Possible values of model parameters}

Wang [2017b] deeply discussed the possible realistic values of model parameters. Only brief explanation is given here. Wang [2017b] stressed that $s \geq 5$ results in stronger coupling, while $s<5$ leads to weaker coupling. In the followings, we consider very weak coupling with $s<1$. Generally, $v_{P}$ is $\sim 10^{-9} \mathrm{~m} / \mathrm{s}$ and thus $V_{P}$ is $\sim 10^{-9}$ when $D_{o} \omega_{0} \approx 1 \mathrm{~m} / \mathrm{sec} . D_{o}$ is almost the maximum displacement, $u_{\max }$, on the ruptured fault plane. The value of $u_{c}$ estimated by Wang [2009] for the $1999 M_{s} 7.6$ Chi-Chi, Taiwan, earthquake is between $3.3 \mathrm{~m}$ for $u_{\max }=10.7 \mathrm{~m}$ and $3.5 \mathrm{~m}$ for $u_{\max }=12.3 \mathrm{~m}$ at shallow depths of the fault zone. This gives $U_{c}=0.30-0.27$. We have $\eta=4 \pi R v \omega_{0} \mathrm{~s} / K$ because of $\eta=C \omega_{0} / L$. In general, $v$ varies from $10^{15} \mathrm{~Pa}$ and $10^{2} \mathrm{~Pa} s$ when the temperature increases from $500^{\circ} \mathrm{C}$ to $1200^{\circ} \mathrm{C}$. Spray [1993] showed $v=10^{3}-10^{6} \mathrm{~Pa} s$ at $800^{\circ} \mathrm{C}$ and $10^{1}-10^{3} \mathrm{~Pa} s$ at $1200^{\circ} \mathrm{C}$. Savage and Lachenbrush [2003] mentioned $v \approx(0.31-3.12) \times 10^{20} \mathrm{~Pa}$ s at a depth of $14 \mathrm{~km}$ of a seismogenic zone. The parameter $R$ is almost the dimension of the deformed volume around a rupture fault. From $2 R=\beta D_{0} / \Delta \sigma$, ( $\beta=$ the shear modulus and $\Delta \sigma=$ stress drop, see Turcotte and Schubert, 1982), we have $R=750$ to $7500 \mathrm{~m}$ when $D_{0}=5 \mathrm{~m}, \Delta \sigma=10-100 \mathrm{MPa}$, and $\beta=30 \mathrm{GPa}$. The average value of $K$ for numerous faults is about $4.6 \times 10^{14} \mathrm{~N} / \mathrm{m}$ [Wang 2012]. Hence, $\eta$ ranges from $10^{-8}$ to $10^{6}$ for $v=10^{2}$ to $10^{15} \mathrm{~Pa} \mathrm{~s}$ when $s<10$ and $\omega_{0}=1 \mathrm{~Hz}$. The time step, $\delta \tau$, can influence simulation results. Practical tests suggest that numerical results are stable when $\delta \tau<0.05$. Hence, $\delta \tau=0.02$ is used in the following computations. In order to kick off slider 1 , an initial normalized force, $\delta F$, is needed. A very small value of $\delta F$ cannot enforce slider 1 to move; while a large one 


\section{Jeen-Hwa Wang}

will dominate computational results. Numerical tests by Carlson et al. [1991] showed that $\delta F=10^{-3}$ is appropriate for numerical simulations. This value is also used hereafter.

\subsection{Results}

Although this study is mainly focused on the inertial effect on interaction between the two sliders, the effects caused by seismic coupling, viscosity, and friction are also taken into account. For each case in study, three diagrams are produced: the time variations in normalized velocity $\left(V / V_{\max }\right)$ and normalized displacement $\left(U / U_{\max }\right)$ and the phase portrait of $V / V_{\max }$ versus $U / U_{\max }$. The values of $V_{\max }$ and $U_{\max }$ for each figure are listed in Table 1. Simulation results are displayed in Figures 3-17 (a solid line for slider 1 and a dotted line for slider 2). The inertial effect between two sliders is absent in Figures 3-9 because of $\mu=1$ and present in Figures 10-15 due to $\mu \neq 1$. In panel (c) of Figures $3-15$, the intersection points of the bisection line (displayed by a dotted line) with the two phase portraits are the fixed points.

The results for seismic coupling effect are displayed in Figure 3 where the values of model parameters are: (a) for $s=0.06$, (b) for $s=0.12$, (c) for $s=0.30$, and (d) for $s=0.48$ when $\mu=1, f_{o 1}=1.0$ and $f_{o 2}=1.0$ (with $\phi=1$ ), $U_{c 1}=0.5$ and $U_{c 2}=0.5$ (with $\psi=1$ ), and $\eta_{1}=0$ and $\eta_{2}=0$ (with $\gamma=1$ ).

\begin{tabular}{|c|c|c|}
\hline Figure Number & $V_{\max }$ & $U_{\max }$ \\
\hline Figure 3 & 0.46320 & 1.35549 \\
\hline Figure 4 & 0.01624 & 0.79366 \\
\hline Figure 5 & 0.01676 & 0.79366 \\
\hline Figure 6 & 0.06563 & 0.98947 \\
\hline Figure 7 & 0.05799 & 0.95699 \\
\hline Figure 8 & 0.02820 & 0.79796 \\
\hline Figure 9 & 0.04886 & 0.79796 \\
\hline Figure 10 & 2.23294 & 1.80950 \\
\hline Figure 11 & 1.01358 & 1.41196 \\
\hline Figure 12 & 1.09037 & 1.80102 \\
\hline Figure 13 & 1.71847 & 1.72252 \\
\hline Figure 14 & 0.55026 & 1.37026 \\
\hline Figure 15 & 0.86796 & 1.71133 \\
\hline Figure 16 & 1.02624 & 1.29862 \\
\hline Figure 17 & 1.44638 & 1.32884 \\
\hline
\end{tabular}

Table 1. The values of $V_{\max }$ and $U_{\max }$ in Figures 3-17. 
The results for frictional effect are displayed in Figures 4-7. The frictional effect includes two factors: the first one from the difference of $f_{01}$ and $f_{02}$ and the second one from the difference in the decreasing rates of dynamic frictional force with increasing displacement, i.e., the difference between $U_{c 1}$ and $U_{c 2}$. Results for the first factor are shown in Figures 4-5. The values of model parameters are: (a) for $\phi=1.00$, (b) for $\phi=1.01$, (c) for $\phi=1.02$, and (d) for $\phi=1.03$ when $s=0.06, \mu=1, f_{o 1}=1.0, U_{c 1}=0.5$ and $U_{c 2}=0.5$ (with $\psi=1$ ), and $\eta_{1}=10$ and $\eta_{2}=10$ (with $\gamma=1$ ) in Figure 4; and (a) for $\phi=1.00$, (b) for $\phi=1.01$, (c) for $\phi=1.02$, and (d) for $\phi=1.03$ when $s=0.48, \mu=1, f_{o 1}=1.0, U_{c 1}=0.5$ and $U_{c 2}=0.5$ (with $\psi=1$ ), and $\eta_{1}=10$ and $\eta_{2}=10$ (with $\gamma=1$ ) in Figure 5. Results for the second factor are shown in Figures 6-7. The values of model parameters are: (a) for $\psi=0.2$, (b) for $\psi=0.6$, (c) for $\psi=1.0$, and (d) for $\psi=1.4$ when $s=0.06, \mu=1, f_{o 1}=1.0$ and $f_{o 2}=1.0$ (with $\phi=1$ ), $U_{c 1}=0.5$, and $\eta_{1}=10$ and $\eta_{2}=10$ (with $\gamma=1$ ) in Figure 6; and (a) for $\psi=0.2$, (b) for $\psi=0.6$, (c) for $\psi=1.0$, and (d) for $\psi=1.4$ when $s=0.48, \mu=1, f_{o 1}=1.0$ and $f_{o 2}=1.0$ (with $\phi=1$ ), $U_{c 1}=0.5$, and $\eta_{1}=10$ and $\eta_{2}=10$ (with $\gamma=1$ ) in Figure 7.

The results for viscous effect are displayed in Figures 8-9. The values of model parameters are: (a) for $\gamma=0.1$, (b) for $\gamma=0.5$, (c) for $\gamma=1.0$, and (d) for $\gamma=1.5$ when $s=0.06, \mu=1, f_{o 1}=1.0$ and $f_{o 2}=1.0$ (with $\phi=1$ ), $U_{c 1}=0.5$ and $U_{c 2}=0.5$ (with $\psi=1$ ), and $\eta_{1}=10$ in Figure 8; and (a) for $\gamma=0.1$, (b) for $\gamma=0.5$, (c) for $\gamma=1.0$, and (d) for $\gamma=1.5$ when $s=0.48, \mu=1, f_{o 1}=1.0$ and $f_{o 2}=1.0$ (with $\phi=1$ ), $U_{c 1}=0.5$ and $U_{c 2}=0.5$ (with $\psi=1$ ), and $\eta_{1}=10$ in Figure 9.

The results for inertial effect are displayed in Figures 10-15. For the six figures, the values of $\mu$ are: (a) for $\mu=0.1$, (b) for $\mu=1$, (c) for $\mu=10$, and (d) for $\mu=50$ when $f_{o 1}=f_{o 2}=1.0$ (with $\phi=1.0$ ) and $\eta_{1}=\eta_{2}=0$. The values of other parameters are: $s=0.12$, and $U_{c 1}=0.5$ and $U_{c 2}=0.2$ (with $\psi=0.4$ ) in Figure 10; $s=0.12$ and $U_{c 1}=0.5$ and $U_{c 2}=0.5$ (with $\psi=1.0$ ) in Figure 11; $s=0.12$ and $U_{c 1}=0.2$ and $U_{c 2}=0.5$ (with $\psi=2.5$ ) in Figure $12 ; s=0.48$ and $U_{c 1}=0.5$ and $U_{c 2}=0.2$ (with $\psi=0.4$ ) in Figure 13; $s=0.48$ and $U_{c 1}=0.5$ and $U_{c 2}=0.5$ (with $\psi=1.0$ ) in Figure $14 ; s=0.48$ and $U_{c 1}=0.2$ and $U_{c 2}=0.5$ (with $\psi=2.5$ ) in Figure 15 .

Figures 16 and 17 are made to examine the effect due to $\phi>1$ for various values of $\mu$. The values of model parameters are: (a) for $\mu=0.1$, (b) for $\mu=1$, (c) for $\mu=10$, and (d) for $\mu=50$ when $f_{o 1}=1.0$ and $f_{o 2}=1.09$ (with $\phi=1.09$ ), $U_{c 1}=U_{c 2}=0.5$ (with $\psi=1$ ), and $\eta_{1}=\eta_{2}=0$. The value of $s$ is 0.12 for Figure 16 and 0.48 for Figure 17 .
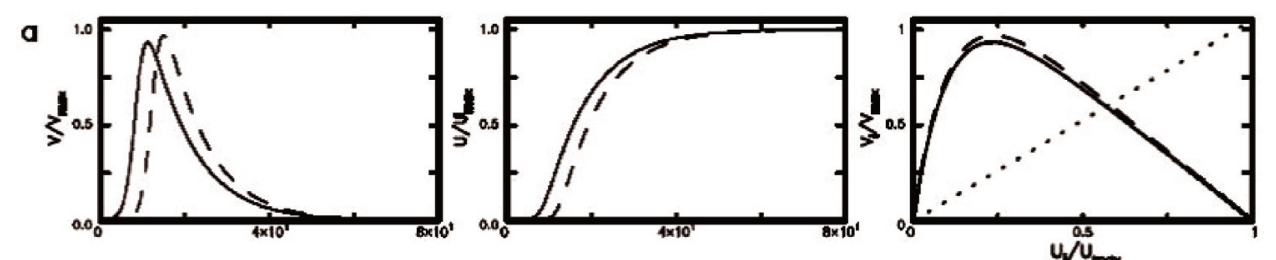

b
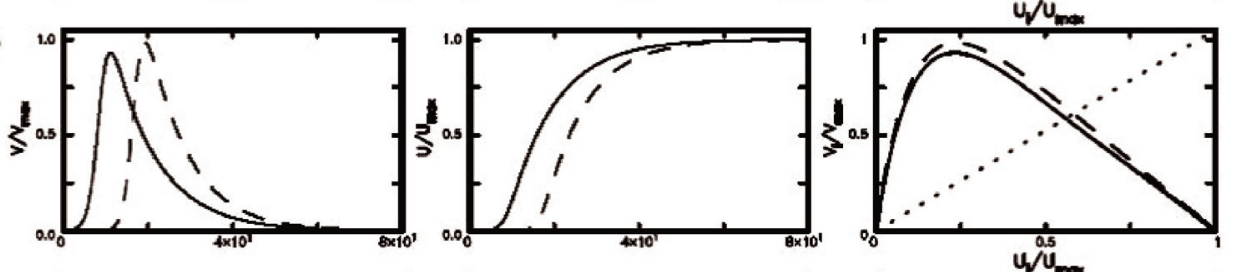

c
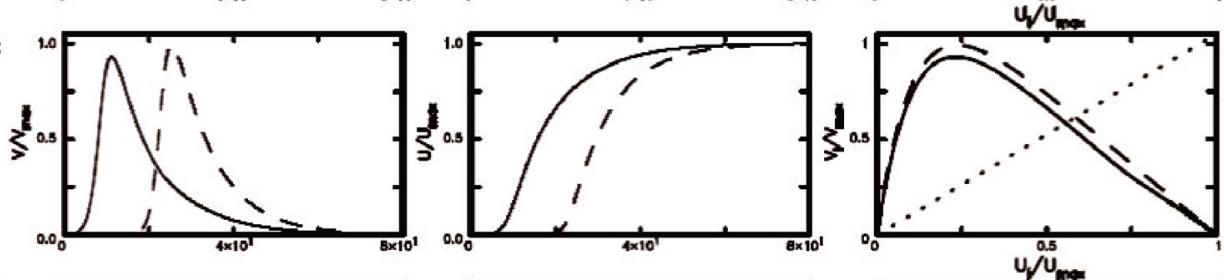

d
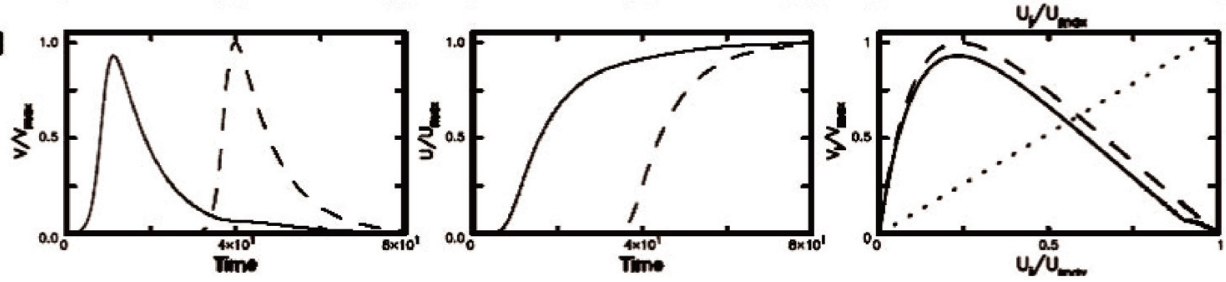

Figure 4. The time sequences of $V / V_{\max }$ and $U / U_{\max }$ and the phase portraits of $V / V_{\max }$ versus $U / U_{\max }$ of the two sliders (solid line for slider 1 and dashed line for slider 2): (a) for $\phi=1.00$, (b) for $\phi=1.01$, (c) for $\phi=1.02$, and (d) for $\phi=1.03$ when $s=0.06, \mu=1, f_{o 1}=1.0, U_{c 1}=0.5$ and $U_{c 2}=0.5$ (with $\psi=1$ ), and $\eta_{1}=10$ and $\eta_{2}=10$ (with $\gamma=1$ ). 
Jeen-Hwa Wang
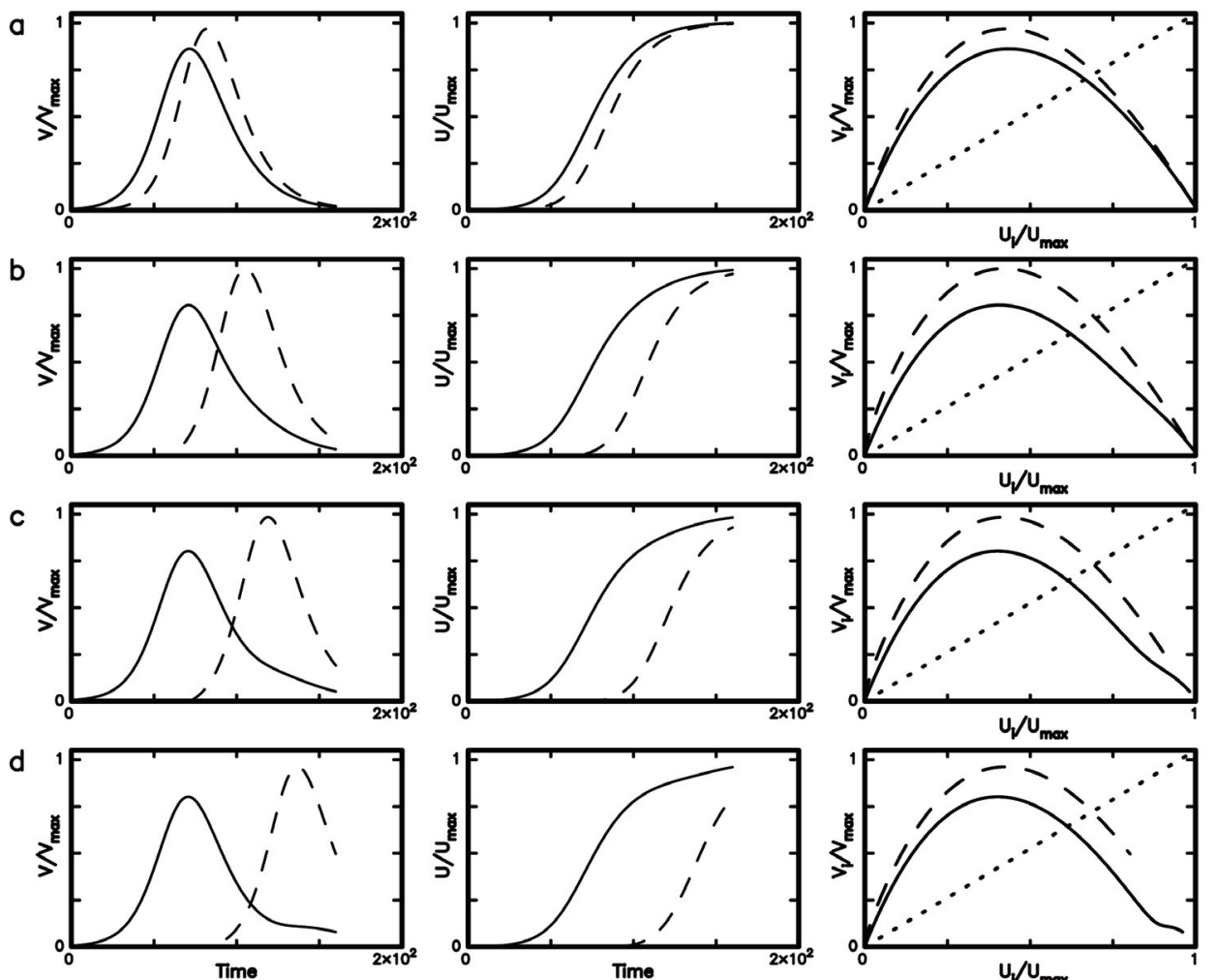

Figure 5. The time sequences of $V / V_{\max }$ and $U / U_{\max }$ and the phase portraits of $V / V_{\max }$ versus $U / U_{\max }$ of the two sliders (solid line for slider 1 and dashed line for slider 2): (a) for $\phi=1.00$, (b) for $\phi=1.01$, (c) for $\phi=1.02$, and (d) for $\phi=1.03$ when $s=0.48, \mu=1, f_{o 1}=1.0, U_{c 1}=0.5$ and $U_{c 2}=0.5$ (with $\psi=1$ ), and $\eta_{1}=10$ and $\eta_{2}=10$ (with $\gamma=1$ ).

a

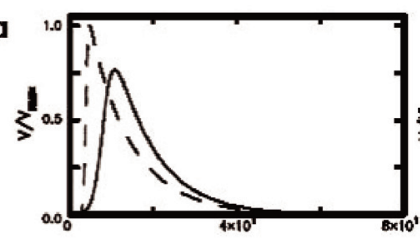

b

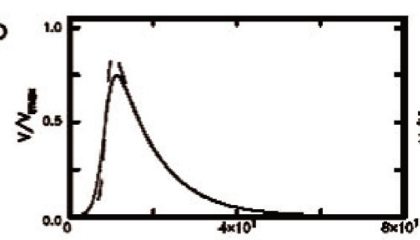

c

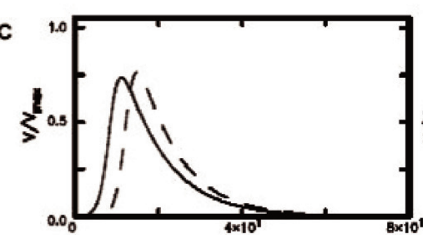

d

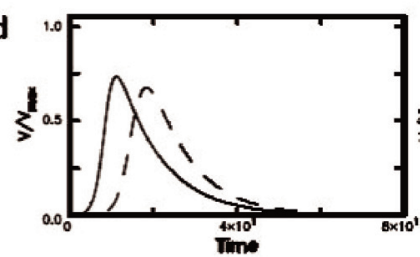

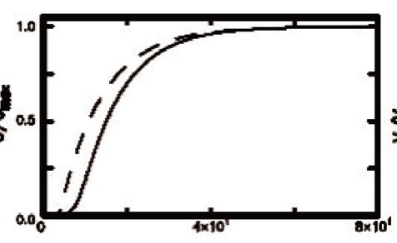
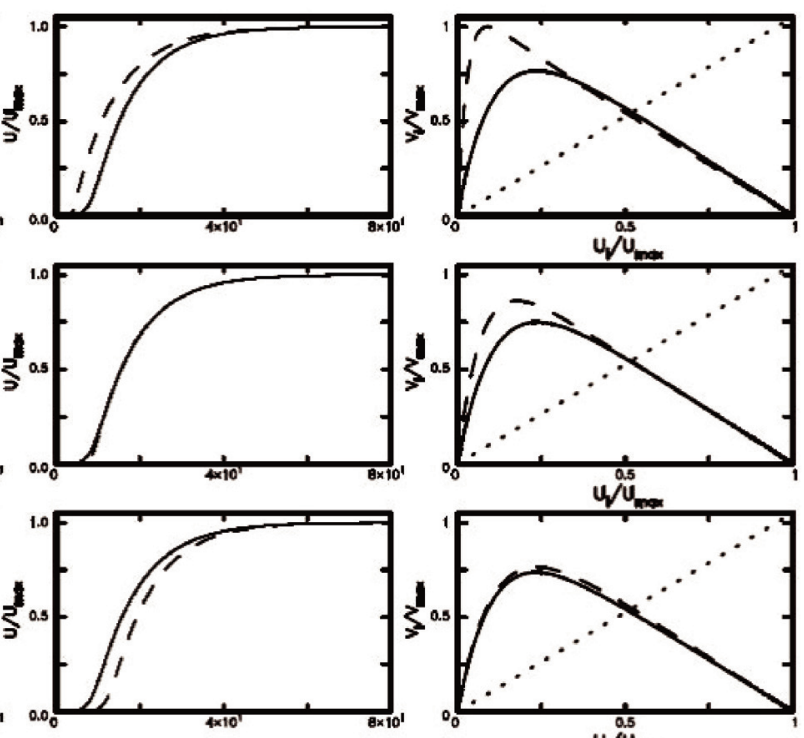

$4 \mathrm{Nu}_{\operatorname{mox}}$
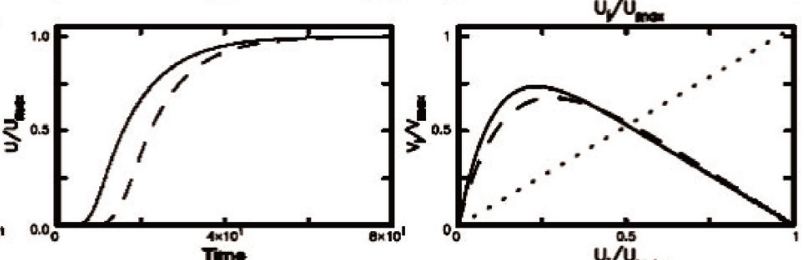

Figure 6. The time sequences of $V / V_{\max }$ and $U / U_{\max }$ and the phase portraits of $V / V_{\max }$ versus $U / U_{\max }$ of the two sliders (solid line for slider 1 and dashed line for slider 2): (a) for $\psi=0.2$, (b) for $\psi=0.6$, (c) for $\psi=1.0$, and (d) for $\psi=1.4$ when $s=0.06, \mu=1, f_{o 1}=1.0$ and $f_{o 2}=1.0$ (with $\phi=1$ ), $U_{c 1}=0.5$, and $\eta_{1}=10$ and $\eta_{2}=10$ (with $\gamma=1$ ). 
a

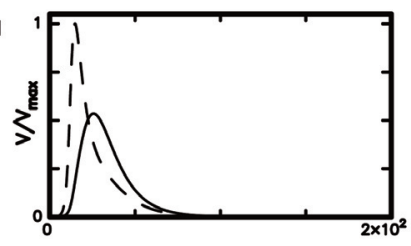

b
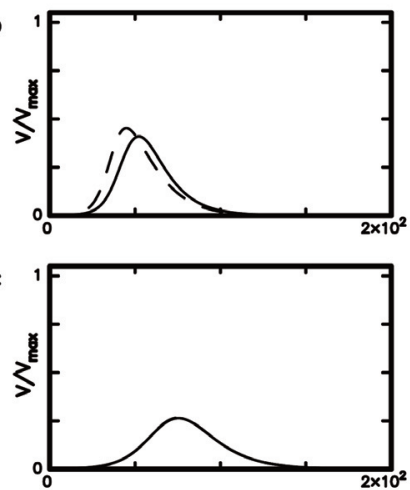

d

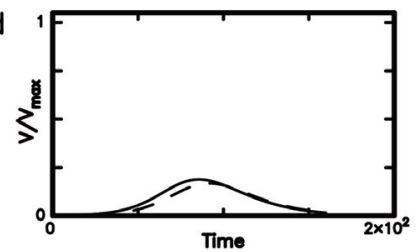

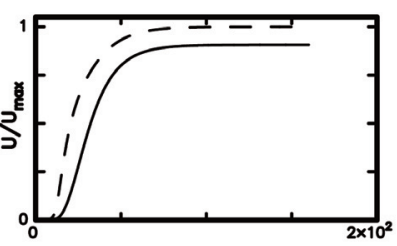
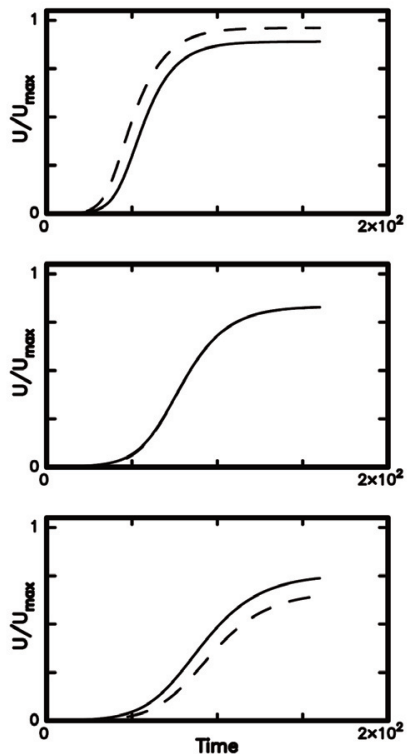
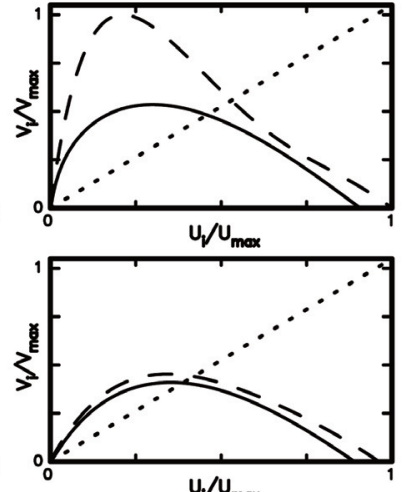

$U_{1} U_{\max }$
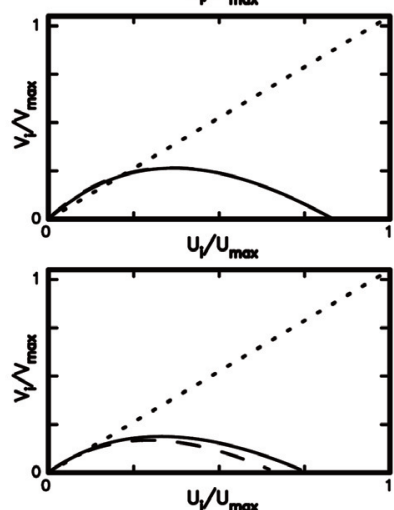

Figure 7. The time sequences of $V / V_{\max }$ and $U / U_{\max }$ and the phase portraits of $V / V_{\max }$ versus $U / U_{\max }$ of the two sliders (solid line for slider 1 and dashed line for slider 2): (a) for $\psi=0.2$, (b) for $\psi=0.6$, (c) for $\psi=1.0$, and (d) for $\psi=1.4$ when $s=0.48, \mu=1, f_{o 1}=1.0$ and $f_{o 2}=1.0$ (with $\phi=1$ ), $U_{c 1}=0.5$, and $\eta_{1}=10$ and $\eta_{2}=10$ (with $\gamma=1$ ).
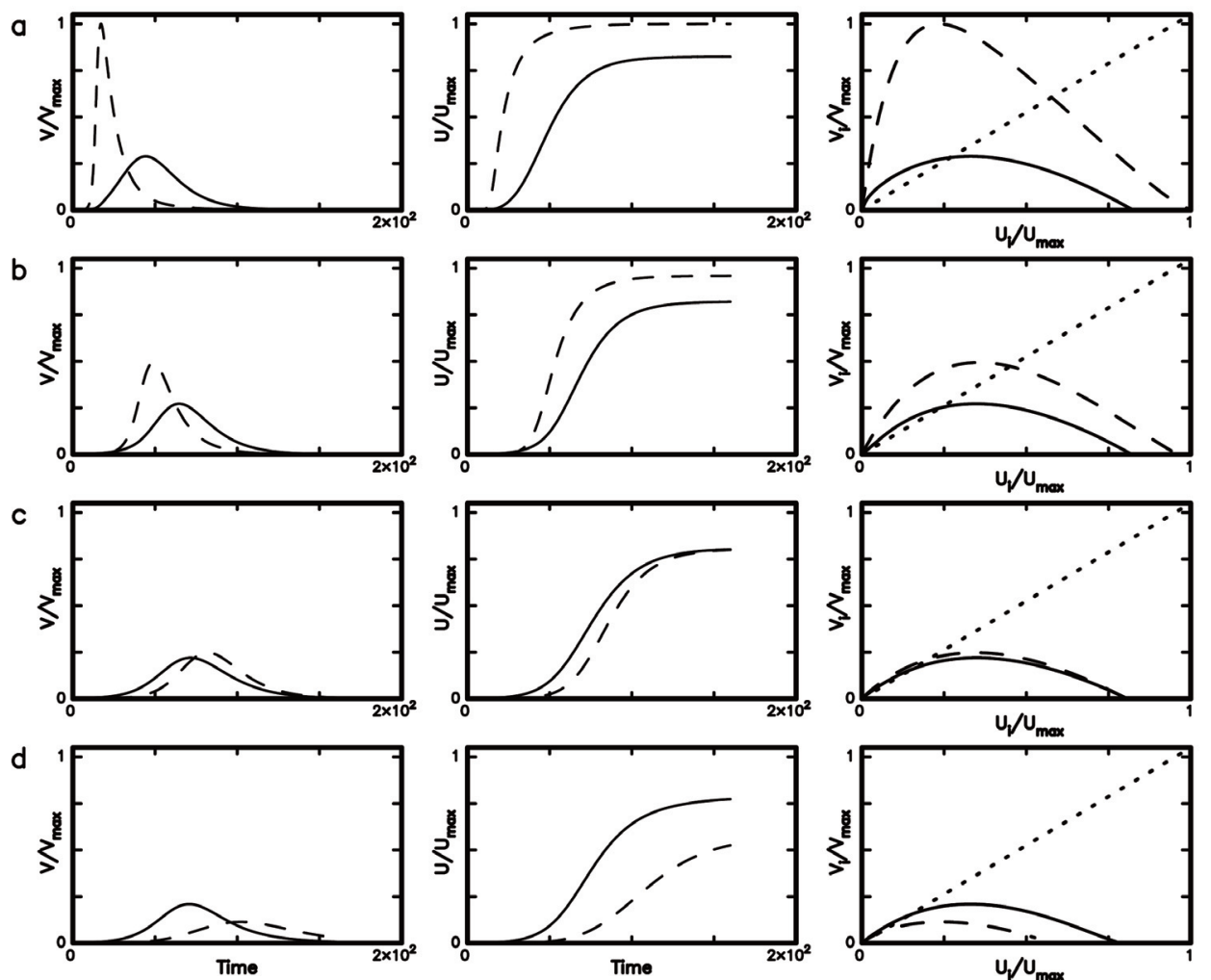

Figure 8. The time sequences of $V / V_{\max }$ and $U / U_{\max }$ and the phase portraits of $V / V_{\max }$ versus $U / U_{\max }$ of the two sliders (solid line for slider 1 and dashed line for slider 2): (a) for $\gamma=0.1$, (b) for $\gamma=0.5$, (c) for $\gamma=1.0$, and (d) for $\gamma=1.5$ when $s=0.06, \mu=1, f_{o 1}=1.0$ and $f_{o 2}=1.0$ (with $\phi=1$ ), $U_{c 1}=0.5$ and $U_{c 2}=0.5$ (with $\psi=1$ ), and $\eta_{1}=10$. 


\section{Jeen-Hwa Wang}

\section{Discussion}

Although this study is focused on the inertial effect on the interaction between two earthquakes, the effects due to seismic coupling, friction, and viscosity are also described below.

\subsection{Seismic coupling effect}

In each plot of Figure 3, the solid line separates from the dashed line, and the difference reduces with increasing $s$, thus showing stronger coupling between the two sliders for larger $s$ than for smaller $s$. Although interaction between the two sliders is weak when $s<1$, slider 2 can still be triggered soon by slider 1 and then move almost simultaneously with slider 1, especially for those with $f_{o 1}=f_{o 2}$. This means that the $s$ is a minor factor in causing time delay on slider 2. In the phase portraits, there are two fixed points: one at the zero point and the other at a non-zero point. The absolute values of slope at the two kinds of fixed points are both larger than 1 and thus they cannot be an attractor. Together with Wang [2017b], the results suggest the importance of $s$ in controlling interaction between two sliders.

\subsection{Frictional Effect}

The frictional effects are caused by two factors: the first one for the difference between $f_{01}$ and $f_{02}$; and the second one for the difference between $U_{c 1}$ and $U_{c 2}$. First, we examine the effect due to the first factor represented by $\phi=f_{o 2} / f_{o 1}$. Since slider 1 is assumed to move before slider 2 does, $\phi$ must be equal to or larger than 1 . Simulation results for $\phi=1.00,1.01,1.02$, and 1.03 when $f_{o 1}=1.0$ are displayed in Figure 4 for $s=0.06$ and Figure 5 for $s=0.48$. In the two figures, the values of other model parameters are: $U_{c 1}=0.5$ and $U_{c 2}=0.5$ (i.e., $\psi=1$ ) and $\eta_{1}=10$ and $\eta_{2}=10$ (i.e., $\gamma=1$ ).
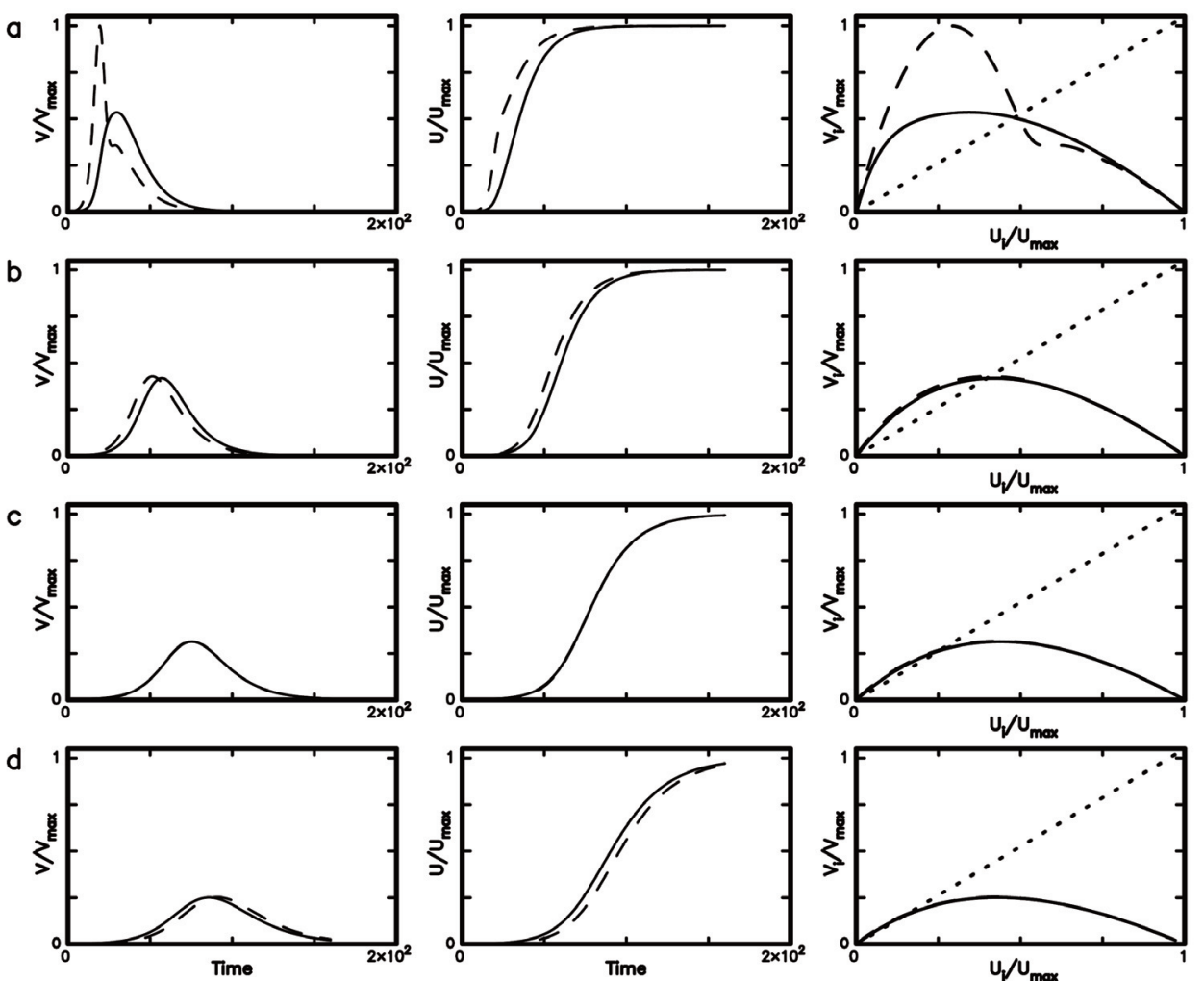

Figure 9. The time sequences of $V / V_{\max }$ and $U / U_{\max }$ and the phase portraits of $V / V_{\max }$ versus $U / U_{\max }$ of the two sliders (solid line for slider 1 and dashed line for slider 2): (a) for $\gamma=0.1$, (b) for $\gamma=0.5$, (c) for $\gamma=1.0$, and (d) for $\gamma=1.5$ when $s=0.48, \mu=1, f_{o 1}=1.0$ and $f_{o 2}=1.0$ (with $\phi=1$ ), $U_{c 1}=0.5$ and $U_{c 2}=0.5$ (with $\psi=1$ ), and $\eta_{1}=10$. 
From Table 1, the values of $V_{\max }$ and $U_{\max }$ have small differences between Figure 4 and Figure 5, thus suggesting that a change of $s$ only yields a small effect on the displacements of sliders. In each plot the solid line is separated from the dashed line and the peak values of $V / V_{\max }$ and $U / U_{\max }$ on slider 2 appear behind those on slider 2 . The difference in the occurrence times of the peak values between the two sliders increases with and decreases with increasing $s$. In other words, higher $f_{02}$ will make a longer delay time on slider 2 than lower $f_{02}$. This is reasonable and suggests that $\phi=f_{02} / f_{01}$ is a significant factor in causing time delay for slider 2 to move after it was triggered by slider 1. Such a difference in the occurrence times is larger in Figure 4 than in Figure 5. This means that higher $s$ leads to stronger coupling, thus yielding a smaller difference in the occurrence times of the peak values between the two sliders than lower $s$. Numerical tests show that slider 2 cannot slide when $\phi \geq 1.05$ for the two values of $s$. This indicates that $\phi=1.05$ is the upper-bound value for the present model. Wang [2017b] obtained that $\phi=1.9$ is the upper-bound value for his model with $s \geq 1$. Hence, the upper-bound value of $\phi$ depends upon the values of other model parameters and could increase with $s$. Results indicate that it is earlier to trigger slider 2 by slider 1 for strong coupling than for weak coupling between the two siders when $\phi>1$. The amplitudes of $V / V_{\max }$ and $U / U_{\max }$ on slider 2 are all larger than those on slider 1, thus suggesting the directivity of motions of the system from slider 1 to slider 2 . A comparison between Figure 4 and Figure 5 shows that the difference in the amplitudes between the two sliders slightly increases with $s$, thus indicating that stronger coupling between the two sliders will produce slightly larger amplitudes on slider 2 than weaker coupling. The predominant periods of the two sliders are almost the same, because the values of model parameters, except for $s$, on the two sliders are equal.

In the phase portraits, there are two fixed points: one at the zero point and the other at a non-zero point. The absolute values of slope at the two kinds of fixed points are both larger than 1 and thus they cannot be an attractor.

Secondly, we examine the effect due to the second factor represented by $\psi=U_{c 1} / U_{c 2}$. Simulation results for $\psi=0.2$, $0.6,1.0$, and $\psi=1.4$ when $U_{c 1}=0.5$ are shown in Figure 6 for $s=0.06$ and Figure 7 for $s=0.48$. In the two figures, the values of other model parameters are: $f_{o 1}=1.0$ and $f_{o 2}=1.0$ (with $\phi=1$ ) and $\eta_{1}=10$ and $\eta_{2}=10$ (with $\gamma=1$ ). From Table 1 , the values of $V_{\max }$ and $U_{\max }$ are slightly higher in Figure 6 than in Figure 7, thus suggesting that the seismic coupling
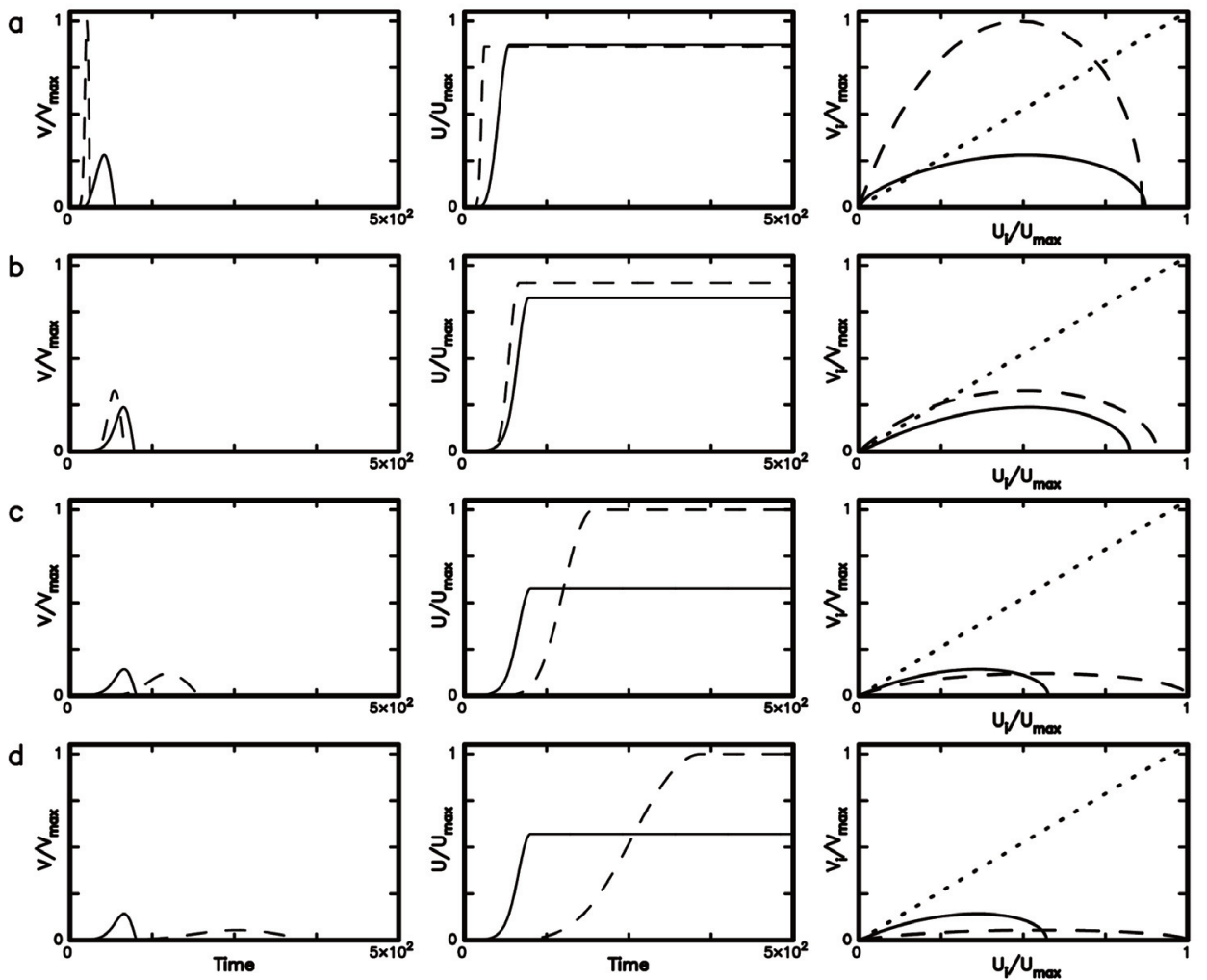

Figure 10. The time sequences of $V / V_{\max }$ and $U / U_{\max }$ and the phase portraits of $V / V_{\max }$ versus $U / U_{\max }$ of the two sliders (solid line for slider 1 and dashed line for slider 2): (a) for $\mu=0.1$, (b) for $\mu=1$, (c) for $\mu=10$, and (d) for $\mu=50$ when $s=0.12, f_{o 1}=f_{o 2}=1.0$ (with $\phi=1$ ), $U_{c 1}=0.5$ and $U_{c 2}=0.2$ (with $\psi=0.4$ ), and $\eta_{1}=\eta_{2}=0$. 
Jeen-Hwa Wang
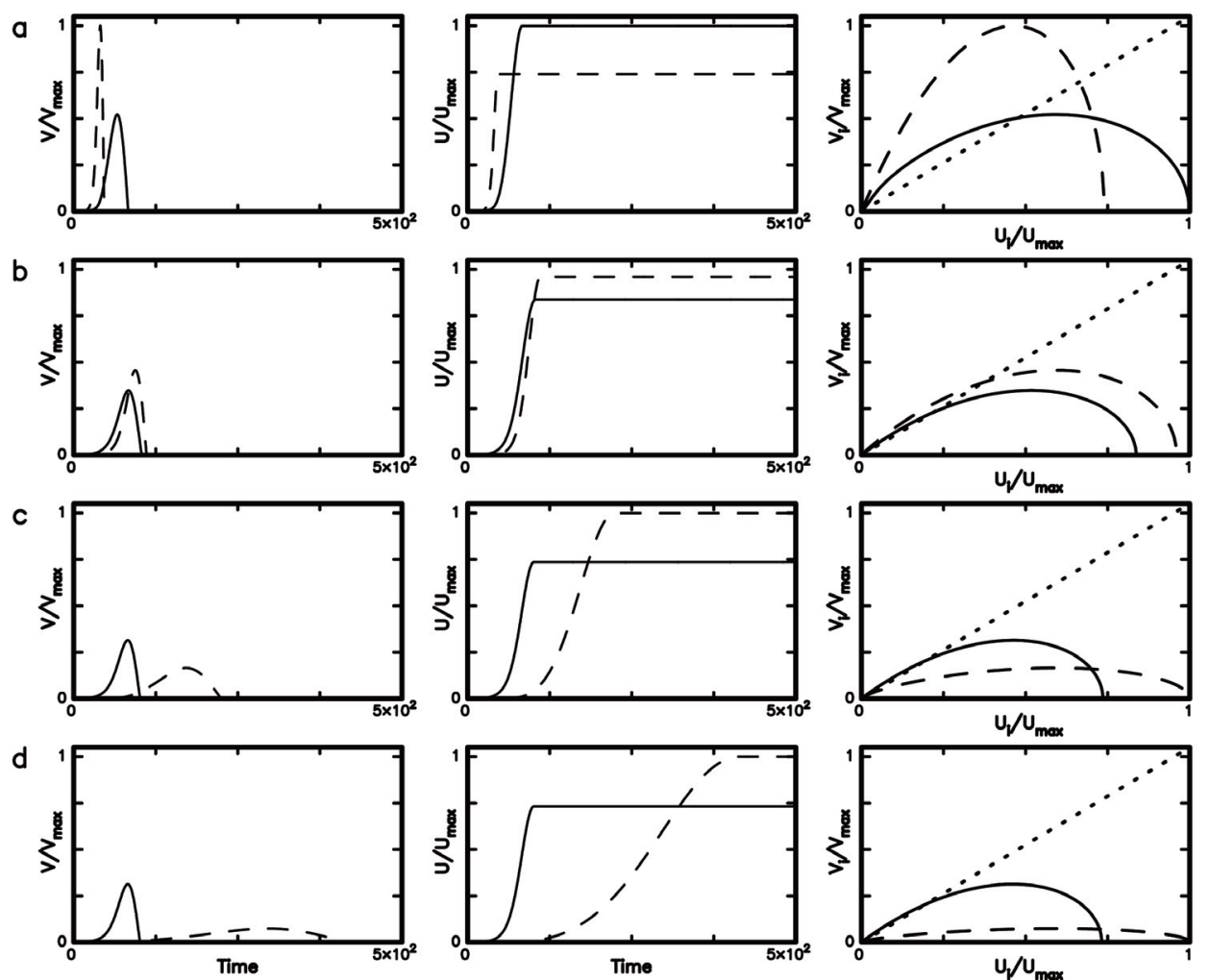

Figure 11. The time sequences of $V / V_{\max }$ and $U / U_{\max }$ and the phase portraits of $V / V_{\max }$ versus $U / U_{\max }$ of the two sliders (solid line for slider 1 and dashed line for slider 2): (a) for $\mu=0.1$, (b) for $\mu=1$, (c) for $\mu=10$, and (d) for $\mu=50$ when $s=0.12, f_{o 1}=f_{o 2}=1.0$ (with $\phi=1$ ), $U_{c 1}=0.5$ and $U_{c 2}=0.5$ (with $\psi=1.0$ ), and $\eta_{1}=\eta_{2}=0$.
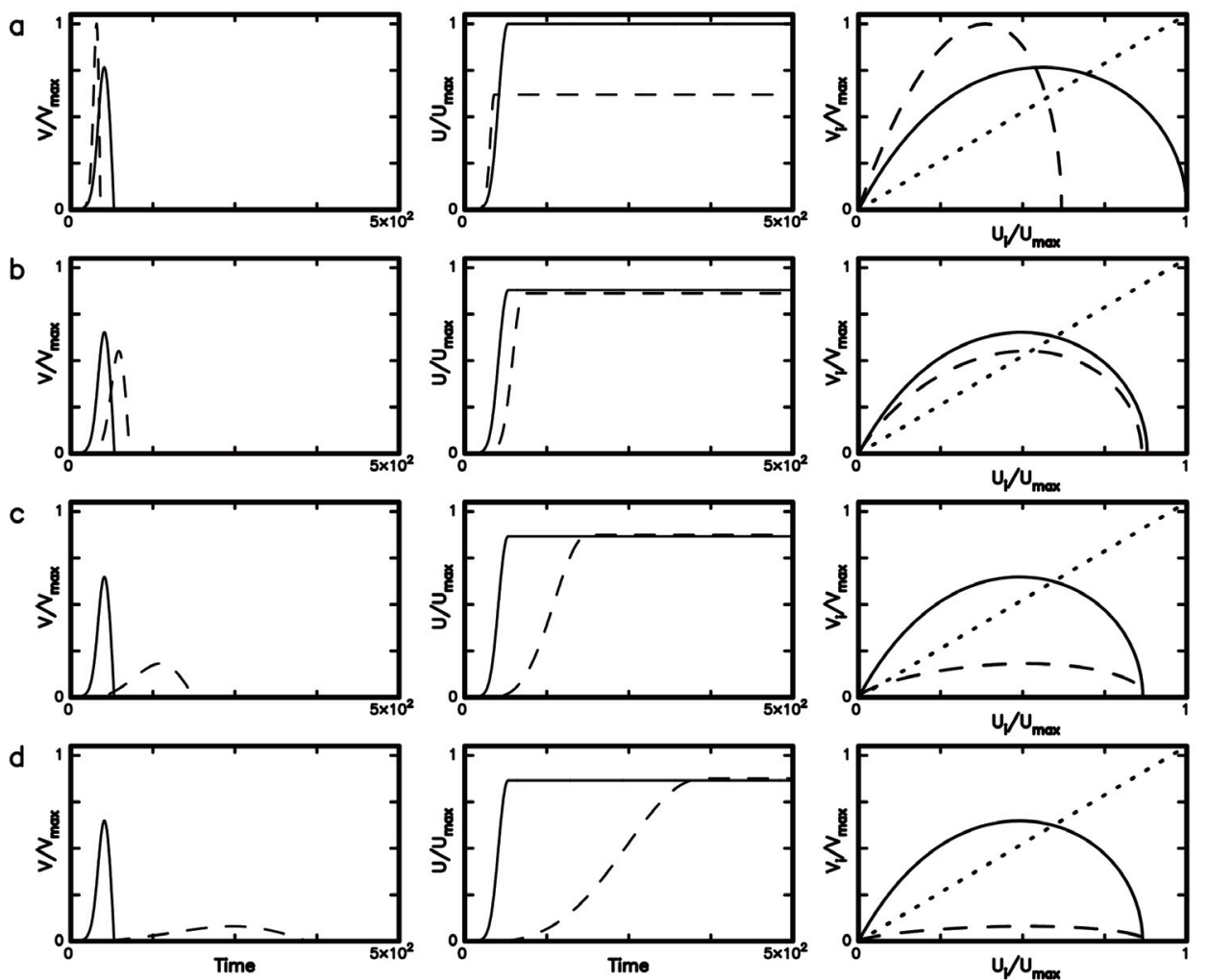

Figure 12. The time sequences of $V / V_{\max }$ and $U / U_{\max }$ and the phase portraits of $V / V_{\max }$ versus $U / U_{\max }$ of the two sliders (solid line for slider 1 and dashed line for slider 2): (a) for $\mu=0.1$, (b) for $\mu=1$, (c) for $\mu=10$, and (d) for $\mu=50$ when $s=0.12, f_{o 1}=f_{o 2}=1.0$ (with $\phi=1$ ), $U_{c 1}=0.2$ and $U_{c 2}=0.5$ (with $\psi=2.5$ ), and $\eta_{1}=\eta_{2}=0$. 


\section{Inertial Effect on Interaction between Two Faults}

only yields a small effect on the displacements of sliders for different values of $\psi$. In each plot the solid line separates from the dashed line, thus exhibiting an effect on slip of and interaction between the two sliders due to a difference of $U_{c 1}$ and $U_{c 2}$. Although slider 1 moves first, the peak values of $V / V_{\max }$ appear earlier on slider 2 than on slider 1 when $\psi<1$, and vice versa when $\psi>1$. This means that the $\psi$ is a significant factor in influencing the movement of slider 2 after it was triggered by slider 1 . Of course, its importance is lower than $\phi$. The peak values of $V / V_{\max }$ and $U / U_{\max }$ of slider 1 are all lower than those of slider 2 when $\psi \leq 1$, and vice versa when $\psi>1$. Results suggest that the condition of $\psi<1$ makes slider 2 be more easily triggered by slider 1 .

In the phase portraits of Figures 6 and 7, the fixed points at the zero point are not an attractor because their absolute values of slope are all higher than 1. The non-zero fixed points in Figure 6 are not an attractor, because their absolute values of slope are higher than 1 . In Figure 7, the non-zero-fixed point for slider 1 when $\psi=1.00$ and those for the two sliders when $\psi=1.01$ are an attractor because the absolute values of slope are lower than 1 . The non-zero fixed points for the two sliders do not exist when $\psi>1.01$. Hence, chaotic behavior may exist under some values of model parameters when $U_{c 2} \neq U_{c 2}$.

Figures 3-7 show that nonlinear slip-dependent friction can result in chaotic slip in the model. This is similar to the chaotic motions in a two-body system studied by others [e.g., Huang and Turcotte 1990, 1992; de Sousa Viera 1999; and Abe and Kato 2013]. A comparison between the cases without viscosity (see Figure 3) and those with viscosity (see Figure 4-10) shows that the absence of viscosity results in higher velocities of sliders than the presence of viscosity, thus suggesting that viscosity can cause slow earthquakes or creep of faults.

\subsection{Viscous effect}

The viscous effect is caused by the difference between $\eta_{1}$ and $\eta_{2}$ and is represented by $\gamma=\eta_{2} / \eta_{1}$. Simulation results for $\gamma=0.1,0.5,1.0$, and 1.5 when $\eta_{1}=10$ are shown in Figure 8 for $s=0.06$ and in Figure 9 for $s=0.48$. The values of other model parameters are $f_{o 1}=1.0$ and $f_{o 2}=1.0$ (with $\phi=1$ ) and $U_{c 1}=0.5$ and $U_{c 2}=0.5$ (with $\psi=1$ ). From Table 1, the value of $V_{\max }$ is slightly higher in Figure 8 than in Figure 9; while that of $U_{\max }$ is almost the same as in the two figures. This suggests that the $s$ yields a slightly larger effect on the velocity than on the displacement for different values of $\psi$.

In each plot the solid line separates from the dashed line, thus exhibiting an effect on slip of the two sliders due to the difference of $\eta_{1}$ and $\eta_{2}$. The peak values of $V / V_{\max }$ of slider 2 appear earlier than those of slider 1 when $\gamma<1$, and vice versa when $\gamma>1$. Hence, the value of $\gamma$ is a significant factor in influencing the occurrence time for slider 2 after it was triggered by slider 1 . The peak values of $V / V_{\max }$ and $U / U_{\max }$ of slider 1 are lower than those of slider 2 when $\gamma<1$, and vice versa when $\gamma>1$.

In the phase portraits of Figures 8 and 9, the fixed points at the zero point are not an attractor because their absolute values of slope are larger than or equal to 1. In Figure 8, the non-zero fixed points for slider 1 are an attractor when $\gamma \leq$, because their slope values are lower than 1 ; while the non-zero fixed point does not exist when $\gamma>1$. In Figure 8, the situation of non-zero fixed point for slider 2 is distinct for different values of $\gamma$. It is not an attractor when $\gamma=0.1$, because its absolute value of slope is higher than 1 ; it is an attractor when $\gamma=0.5$, because its absolute value of slope is lower than 1 ; and the non-zero fixed point does not exist when $\gamma>0.5$. In Figure 9, the nonzero fixed points for the two sliders are not an attractor, because their absolute values of slope are higher than 1 . The non-zero fixed points for slider 1 are an attractor when $\gamma \leq 1.0$, because their absolute values of slope are lower than 1 ; while the non-zero fixed point does not exist when $\gamma=1.5$. The non-zero fixed point for slider 2 is not an attractor when $\gamma=0.1$, because its absolute value of slope is higher than 1 ; the non-zero fixed points are an attractor when $\gamma=0.5$ and 1.0, because their absolute value of slope are lower than 1 ; and the non-zero fixed point does not exist when $\gamma=1.5$.

Since the slip starts at slider 1 and then proceeds to slider 2, the epicenter of the related event consisting of slip of the two sliders is at slider 1 and slider 2 shows a ruptured fault away from the epicenter. Results displayed in the plots denoted by "a" in Figures 6-9 seem able to provide a mechanism to interpret the phenomenon that during an event the major slip does not happen at the epicenter and occurs at some other places for numerous earthquakes as addressed by some authors [e.g., Wang 2006; Lee et al. 2011]. When the characteristic displacement, $u_{c}$, of friction law and/or the viscosity coefficient of an area which is not around the epicenter is lower than that at the epicenter, the final slip at that area could be larger than that at epicenter as implied from those plots.

The longer duration times in Figures 8 and 9 than in Figures $3-7$ are due to the viscous effect. When $K=0$, the 


\section{Jeen-Hwa Wang}

normalized natural periods of the two sliders of the model obtained by Wang [2017b] are: (1) $T_{o 1}=2 \pi$ for slider 1 and $T_{02}=2 \pi \mu^{1 / 2}$ for slider 2 in the absence of friction and viscosity; and (2) $T_{1}=T_{01} /\left(4-\eta 1^{2}\right)^{1 / 2}$ for slider 1 and $T_{2}=T_{02} /(4-$ $\left.\eta_{2}^{2}\right)^{1 / 2}$ for slider 2 in the present of viscosity. This gives $T_{2} / T_{1}=\left[\mu\left(4-\eta_{1}^{2}\right) /\left(4-\eta_{2}^{2}\right)\right]^{1 / 2}$.

Obviously, the inertial and viscous effects increase the natural periods. When the two sliders are linked together, the natural period of each slider must be slightly different from $T_{0}$. The difference may be small because of weak coupling (with $s<1$ ) between the two sliders. In Figures 8 and $9, T_{1}>T_{2}$ when $\eta_{1}>\eta_{2}$ (see Figures $8 \mathrm{a}$ and $9 \mathrm{a}$ ), $T_{1}=T_{2}$ when $\eta_{1}=\eta_{2}$ (see Figure $8 \mathrm{~b}$ and $9 \mathrm{~b}$ ), and $T_{1}<T_{2}$ when $\eta_{1}<\eta_{2}$ (see Figures $8 \mathrm{c}, \mathrm{d}$ and $9 \mathrm{c}, \mathrm{d}$ ). The values of $V / V_{\max }$ and $U / U_{\max }$ of slider 2 both decrease with increasing $\eta_{2}$, because viscosity can depress the movements of sliders. Wang [2007] applied a two-body model with viscosity to simulate the difference in ground motions between the northern and southern segments of the Chelungpu fault generated by the $1999 \mathrm{Chi}$-Chi, Taiwan, $M_{s} 7.6$ earthquake. The observed predominant periods at nine near-fault seismic stations are longer in the northern segments than in the southern one. His results show that higher viscosity coefficient in the fault zone of the former than in that of the latter is responsible for the observation.

\subsection{Inertial effect}

The inertial effect (represented by $\mu$ ) is made for different masses of the two sliders, i.e., $\mu \neq 1$. Results are displayed in Figures 10-12 with $s=0.20$ for weaker seismic coupling and in Figures $13-15$ with $s=0.48$ for strong seismic coupling. In the six figures, the values of $\mu$ are: (a) for $\mu=0.1$, (b) for $\mu=1$, (c) for $\mu=10$, and (d) for $\mu=50$, with $U_{c 1}=0.5$ and $U_{c 2}=0.2$ (i.e., $\psi=0.4$ ) in Figures 10 and 13, with $U_{c 1}=0.5$ and $U_{c 2}=0.5$ (i.e., $\psi=1.0$ ) in Figures 11 and 14, and with $U_{c 1}=0.2$ and $U_{c 2}=0.5$ (i.e., $\psi=2.5$ ) in Figures 12 and 15 when $f_{o 1}=f_{o 2}=1.0$ and $\eta_{1}=\eta_{2}=0$. Like Figures 3-9, Figures 10-15 show the triggering of slider 2 by sider 1 . All figures display decreases in the peak velocities and final displacements with increasing $\mu$. This indicates that the condition of slider 2 being heavier than slider 1 can reduce the movements of the two sliders.

In Figures 10-15, the peak velocity and final displacement on slider 2 appear earlier than those on slider 1 when $\mu=0.1$ for both weaker and stronger seismic coupling; the peak velocity and final displacement on slider 2 appear either earlier $(\psi<1.0)$ or later $(\psi \geq 1.0)$ than those on slider 1 when $\mu=1$ for both weaker and stronger seismic coupling; and the peak velocity and final displacement on slider 2 appear later (for weaker seismic coupling with $s=0.12$ ) or earlier (for stronger seismic coupling with $s=0.48$ ) than those on slider 1 when $\mu>1$. This means that in addition to the difference in static friction forces between the two sliders, the inertia is also a factor in delaying the movement of slider 2. In Figures 13-15, during the computational time a few smaller-sized events appear after the first one on slider 1 when $\mu>1$; an event appears after the first one on slider 2 when $\mu<1$; and not any event appears after the first one on the two sliders when $\mu=1$. These phenomena cannot be found in Figures 10-12 with weaker seismic coupling. This means that when the coupling between the two sliders is weak, aftershocks or afterslip cannot be generated on the lighter slider.

In the middle panels of Figures 10-15, the displacement of slider 1 (displayed by a solid line) first appears and increases with time; while the displacement of slider 2 (displayed by a dashed line) suddenly appears for a while after slider 1 moves and then jumps to its peak value in a short time span. The difference in final displacement between the two sliders slightly increases with $\mu$ and is bigger for small $s$ than for large $s$. The phenomenon that the final displacement of slider 1 is lower than that of slider 2 might be due to a higher force drop on slider 2 than on slider 1 .

In order to examine the possible effect caused by $\phi>1$ for various values of $\mu$, numerical results are displayed in Figures 16 and 17 when $\phi=1.09$. Numerical tests show that $\phi=1.09$ and 1.30 are the upper-bounds for $s=0.12$ and 0.48 , respectively, to prohibit the motion of slider 2 . This is consistent with the above-mentioned conclusion that the upper bound of $\phi$ increases with $s$. The two figures exhibit that slider 2 moves later than slider 1 and the difference of starting times of movement on the two sliders increase with $\mu$. A comparison between Figure 11 with $\phi=1$ and Figure 16 with $\phi=1.09$ shows that there is only a small difference between the two panels with $\mu=0.1$ and 1 . On slider 1 there is only an event showed in Figure 11 and numerous small events are generated after the first one in Figure 16. A comparison between Figure 14 with $\phi=1$ and Figure 17 with $\phi=1.09$ shows that there is only a small difference between the two panels when $\mu=0.1$ and 1 . On slider 1 numerous small events are generated after the first one in Figure 17 and there is only an event produced in Figure 14.

In these figures, the predominant period of slider 1 does not change with $\mu$ and that of slider 2 increases with $\mu$ 
a

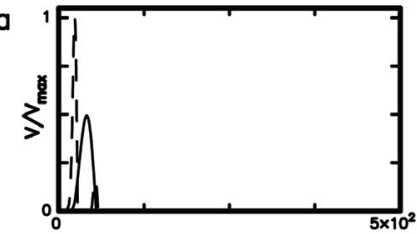

b

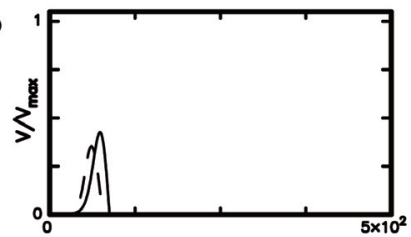

c

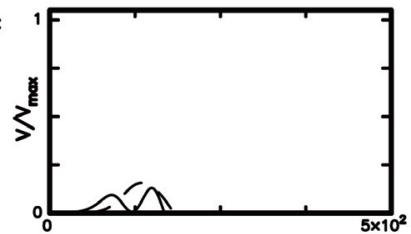

d

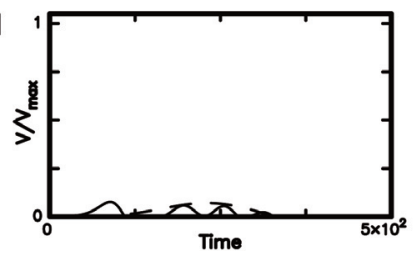

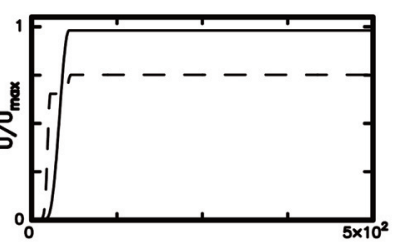
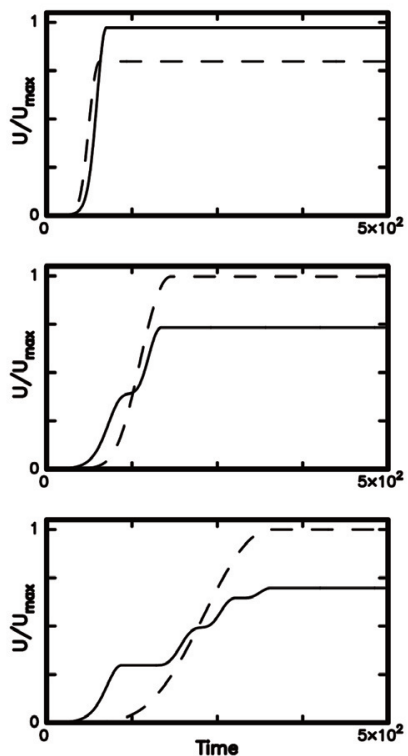
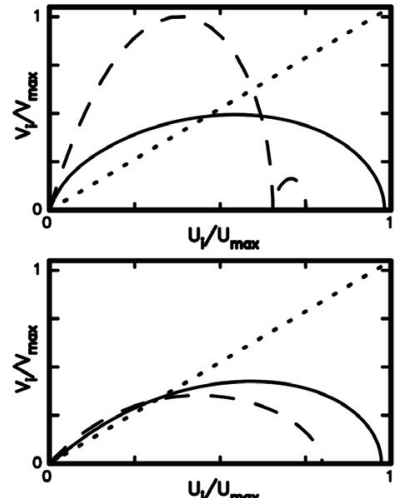

$u_{\sqrt{ }} U_{\max }$

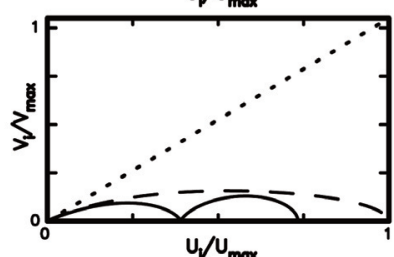

$\mathrm{U}_{\sqrt{ }} \mathrm{U}_{\max }$

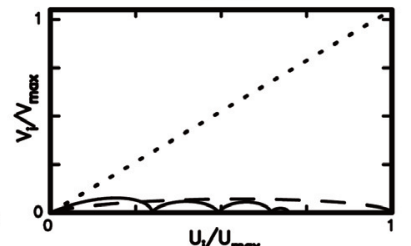

Figure 13. The time sequences of $V / V_{\max }$ and $U / U_{\max }$ and the phase portraits of $V / V_{\max }$ versus $U / U_{\max }$ of the two sliders (solid line for slider 1 and dashed line for slider 2): (a) for $\mu=0.1$, (b) for $\mu=1$, (c) for $\mu=10$, and (d) for $\mu=50$ when $s=0.48, f_{o 1}=f_{o 2}=1.0$ (with $\phi=1$ ), $U_{c 1}=0.5$ and $U_{c 2}=0.2$ (with $\psi=0.4$ ), and $\eta_{1}=\eta_{2}=0$.
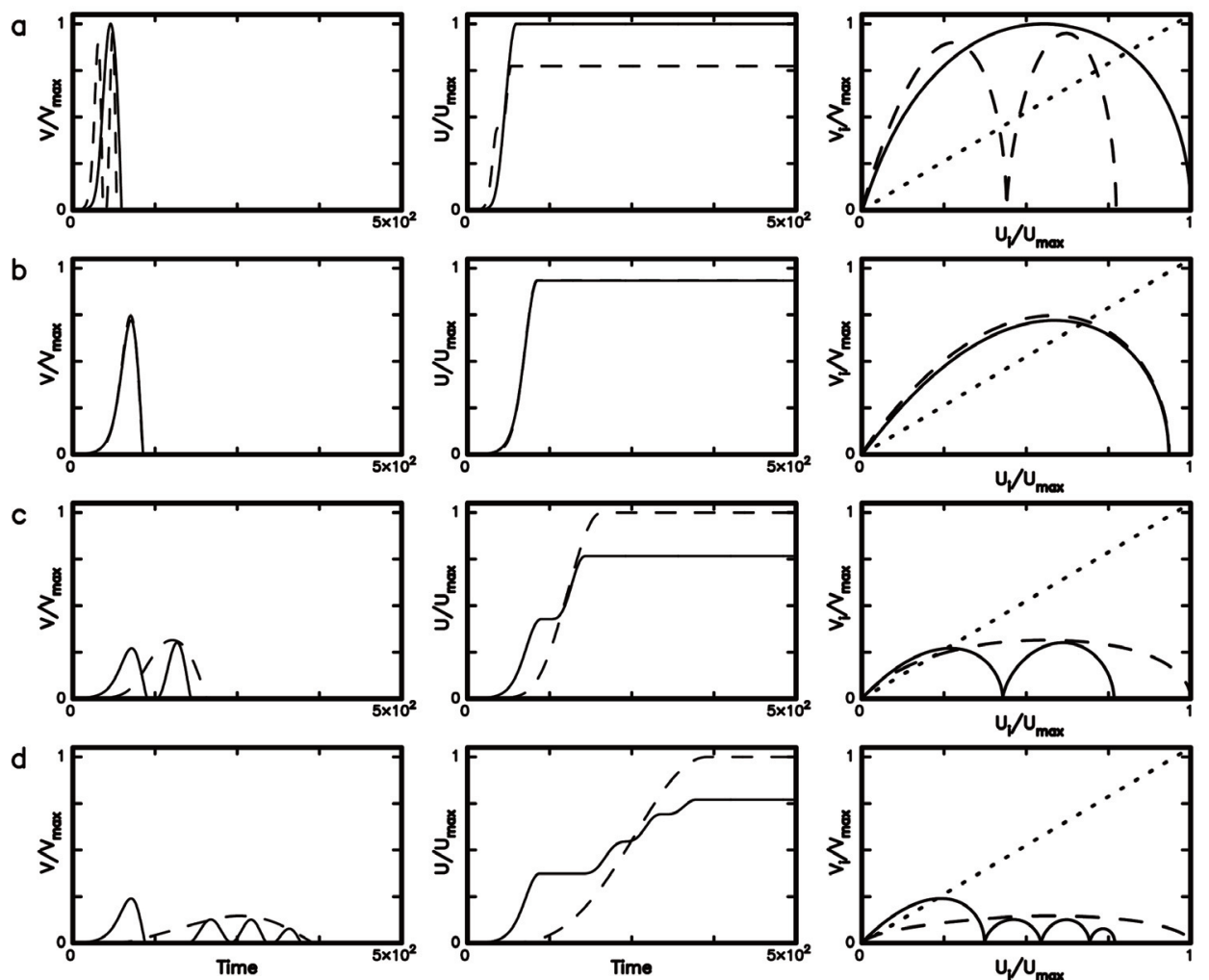

Figure 14. The time sequences of $V / V_{\max }$ and $U / U_{\max }$ and the phase portraits of $V / V_{\max }$ versus $U / U_{\max }$ of the two sliders (solid line for slider 1 and dashed line for slider 2): (a) for $\mu=0.1$, (b) for $\mu=1$, (c) for $\mu=10$, and (d) for $\mu=50$ when $s=0.48, f_{o 1}=f_{o 2}=1.0$ (with $\phi=1$ ), $U_{c 1}=0.5$ and $U_{c 2}=0.5$ (with $\psi=1.0$ ), and $\eta_{1}=\eta_{2}=0$. 


\section{Jeen-Hwa Wang}

as expressed in the above-mentioned equations of $T_{1}$ and $T_{2}$. The peak velocities on both sliders are abnormally large and decrease with increasing $\mu$. From Equation (3b), there is a very large acceleration on slider 2, i.e., $d^{2} U_{2} / d \tau^{2}$, caused by a very small value of $\mu=0.1$. This will lead to an abnormally large velocity. This phenomenon exists for $\mu<1$. As $\mu>1$, the acceleration decreases with increasing $\mu$. As mentioned above, when $\mu=0.1$ the peak velocities on slider 2 in Figures 13-15 are higher, respectively, than those in Figures 10-12. This indicates that that weaker coupling between two sliders can yield a higher peak velocity than stronger coupling when $\mu=0.1$ or $m_{1}=10 m_{2}$. From the six figures we can see that the peak velocities on the two sliders, especially for slider 2 , decrease with increasing $\mu$. In Figures 10-15, when $\mu=0.1$ the final displacements on slider 1 are almost larger than those on slider 2 except for the case in Figure 10 in which the former is only slightly larger than the latter; when $\mu=1$ the final displacements on slider 1 are larger than, equal to, or smaller than those on slider 2 depending on the values of other model parameters; and when $\mu>1$ the final displacements on slider 2 are larger than those on slider 1 except for the cases in Figure 10 where the final displacements on slider 2 are approximately equal to those on slider 1. During the computational time, only one event is generated on each slider when $s=0.12$; on the other hand only one event is generated on slider 2 and numerous smaller-sided events are produced after the first largest one on slider 1 when $s=0.48$.

When $\mu \geq 50$, the predominant period (see panel (a) in each figure) and rise time (see panel (b) in each figure) of slider 2 become very long and much longer than those of slider 1 , such that the slip of slider 2 behaves like a slow earthquake. This suggest that slider 2 cannot behave like a regular earthquake when $\mu \geq 50$ or $m_{2} \geq 50 m_{1}$. When the densities, $\rho$, and fault widths, $W$, of the two sliders are equal, we have $m_{\mathrm{i}}=\rho W L_{i}$, where $L_{i}$ is the fault length of slider $i(i=1,2)$. Since the present model is a strike-slip (SS) one, the empirical relationship of earthquake magnitude, $M$, versus fault length, $L$, for the SS events is (Wells and Coppersmith, 1994): $M=(5.16 \pm 0.13)+(1.12 \pm 0.08) \log (L)$. Consider the magnitude of slider 1 is $M_{1}$. For $\mu=50$ or $L_{2}=50 L_{1}$, the magnitude of slider 2 is $M_{1}+1.90$. This means that an earthquake with a magnitude of $M$ on slider 1 (or fault 1) can trigger an even with a magnitude $<M+1.90$ as $\mu<50$ and can initiate a slow event with a magnitude $\geq M+1.90$ as $\mu \geq 50$ on slider 2 (or fault 2).
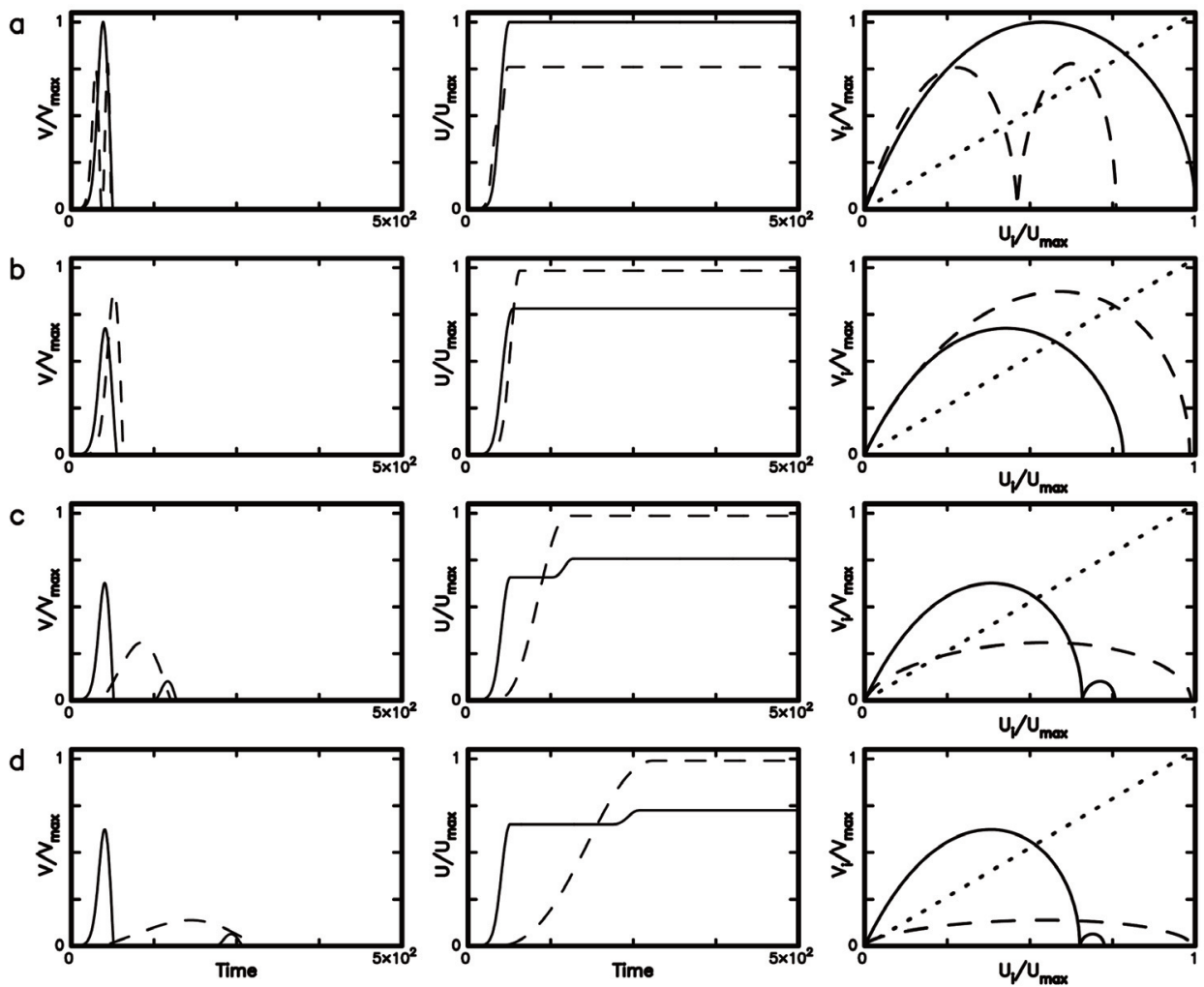

Figure 15. The time sequences of $V / V_{\max }$ and $U / U_{\max }$ and the phase portraits of $V / V_{\max }$ versus $U / U_{\max }$ of the two sliders (solid line for slider 1 and dashed line for slider 2): (a) for $\mu=0.1$, (b) for $\mu=1$, (c) for $\mu=10$, and (d) for $\mu=50$ when $s=0.48, f_{o 1}=f_{o 2}=1.0$ (with $\phi=1$ ), $U_{c 1}=0.2$ and $U_{c 2}=0.5$ (with $\psi=2.5$ ), and $\eta_{1}=\eta_{2}=0$. 
Equation (3) shows that the relative movement between the two sliders may induce an additional force to slider 1 when $U_{2}-U_{1}>0$ and a resistant force to slider 1 when $U_{2}-U_{1}<0$. Hence, a decrease in $U_{2}$ due to an increase in the inertia on slider 2 would resist slider 1 to move, and the resistant force increases with $s$.

In the phase portraits on the right-hand-side panels of Figures 10-17, there are fixed points at either zero or nonzero points for the two sliders. When $\mu<1$, the fixed points are not an attractor because their absolute values of slope are larger than or equal to 1 . When $\mu=1$, the fixed points at the zero points for slider 2 could be an attractor because their absolute values of slope are smaller than 1 for $\psi \leq 1$ and cannot be an attractor because their absolute values of slope are larger than 1 for $\psi>1$. There are not fixed points at non-zero points when $\mu \leq 1$. In Figures 10-15 with $\mu>1$, the fixed points at either the zero or non-zero points for the two sliders are not an attractor because their absolute values of slope are larger than or equal to 1 . In Figures 16 and 17 with $\mu>1$, there is only a fixed point at the zero point and there is not a fixed point at the non-zero point for the two sliders. The fixed point at the zero point could be an attractor because their absolute values of slope are smaller than 1 . Consequently, the inertia can influence the chaotic behavior of the system.

In addition, Figures $10-15$ with $s=0.48$ also exhibit three interesting and significant features: First, the mainshock occurs on the heavier slider (having a longer fault) which is slider 1 for $\mu<1$ and slider 2 for $\mu>1$. Secondly, when $\mu<1$ a few smaller-sized events, which could be regarded as aftershocks, are generated on the lighter slider (having a shorter fault), i.e., slider 2 . Thirdly, when $\mu>1$ a few smaller- sized events, which could be regarded as foreshocks and aftershocks, are generated on the lighter slider (having a shorter fault), i.e., slider 1 now. The three features cannot be found in the figures with $s=0.12$ even $\mu>>1$. Results seem to suggest that the mainshock occurs on the heavier slider and foreshocks and aftershocks are generated on the lighter slider when seismic coupling is stronger; while only the mainshock is produced when seismic coupling is weaker. Since the present model is still simple, simulation results cannot give us comprehensive information about the generation of a whole foreshocksmainshock-aftershocks sequence. A more comprehensive model is necessary for this purpose.

a

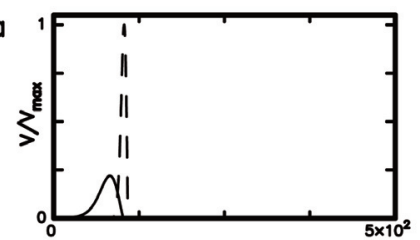

b

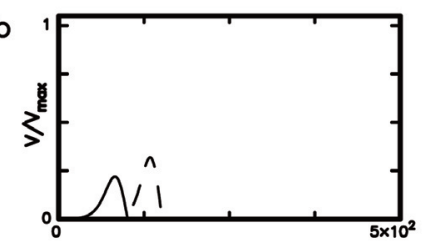

c

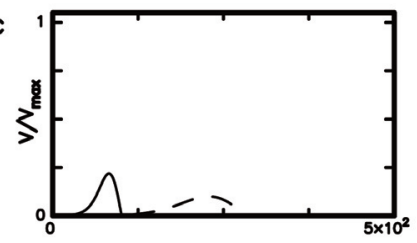

d

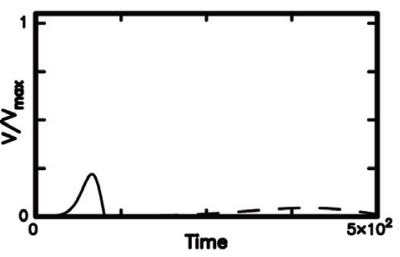

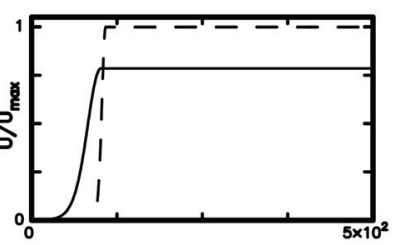
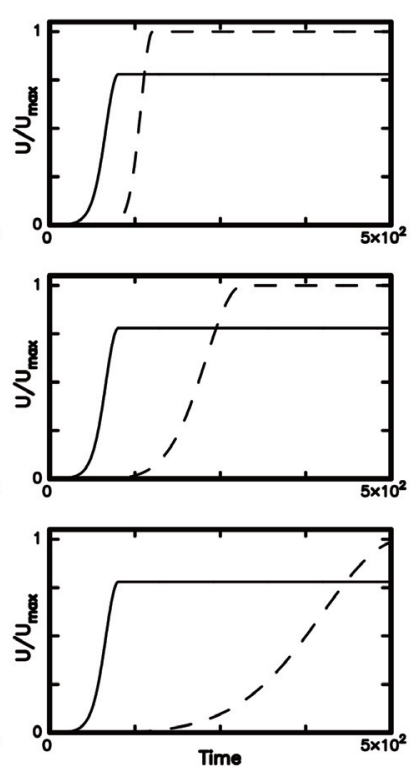
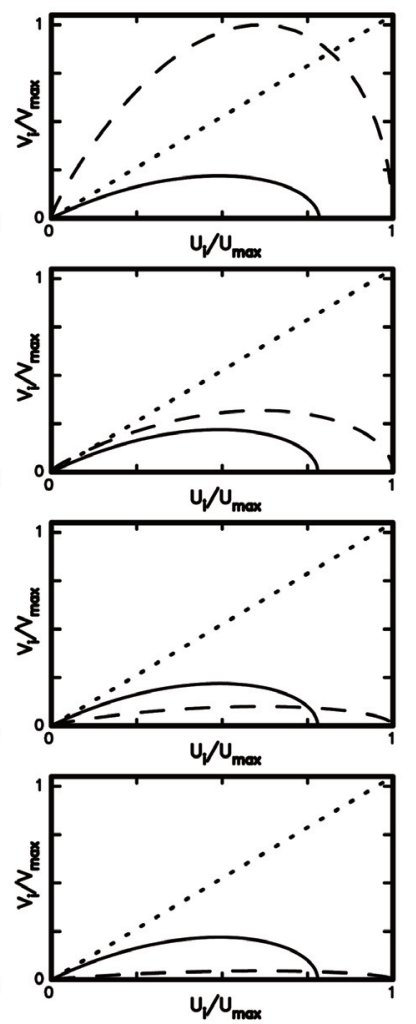

Figure 16. The time sequences of $V / V_{\max }$ and $U / U_{\max }$ and the phase portraits of $V / V_{\max }$ versus $U / U_{\max }$ of the two sliders (solid line for slider 1 and dashed line for slider 2): (a) for $\mu=0.1$, (b) for $\mu=1$, (c) for $\mu=10$, and (d) for $\mu=50$ when $s=0.12, f_{o 1}=1.00$ and $f_{o 2}=1.09$ (with $\phi=1.09$ ), $U_{c 1}=U_{c 2}=0.5$ (with $\psi=1$ ), and $\eta_{1}=\eta_{2}=0$. 


\section{Jeen-Hwa Wang}

\section{Conclusion}

Simulation results show that although the stiffness ratio $s$ can control the interaction between the two sliders, it is only a minor factor in causing time delay between the two sliders. The difference between $f_{o 2}$ and $f_{o 1}$, which is represented by $\phi=f_{02} / f_{o 1}$, is a significant factor in causing time delay of the motion of slider 2 after it was triggered by slider 1 . Higher $\phi$ leads to a longer delay time than lower $\phi$. Slider 2 cannot move when $\phi$ is higher than a critical value which depends on other model parameters. Interaction between the two sliders is different between $\psi=U_{c 2} / U_{c 1}<1$ and $\psi>1$, and thus $\psi$ is also a significant factor in causing time delay for slider 2 to move triggered by slider 1 . Of course, it is less important than $\phi$. The slip patterns of two sliders yielded by small $\psi$ are opposite to those done by large $\psi$. The differences in amplitudes and particle velocities between the two sliders decrease with increasing $\gamma\left(=\eta_{2} / \eta_{1}\right)$. The $\gamma$ is a significant factor in causing time delay for slider 2 to move triggered by slider 1 . Higher results in a longer delay time than lower $\gamma$. The presence of viscosity can make an increase in the duration time and predominant period of motions of a slider and may generate attractors for some values of $\gamma$. The inertial effect denoted by $\mu=m_{2} / m_{1}$ plays a significant role on the interaction between the two sliders (or two faults). Foreshocks or aftershocks/afterslip can be generated on slider 1 or slider 2 when $\mu \neq 1$. The slip of slider 2 behaves like a slow event when $\mu>50$ or $m_{2}>50 m_{1}$.
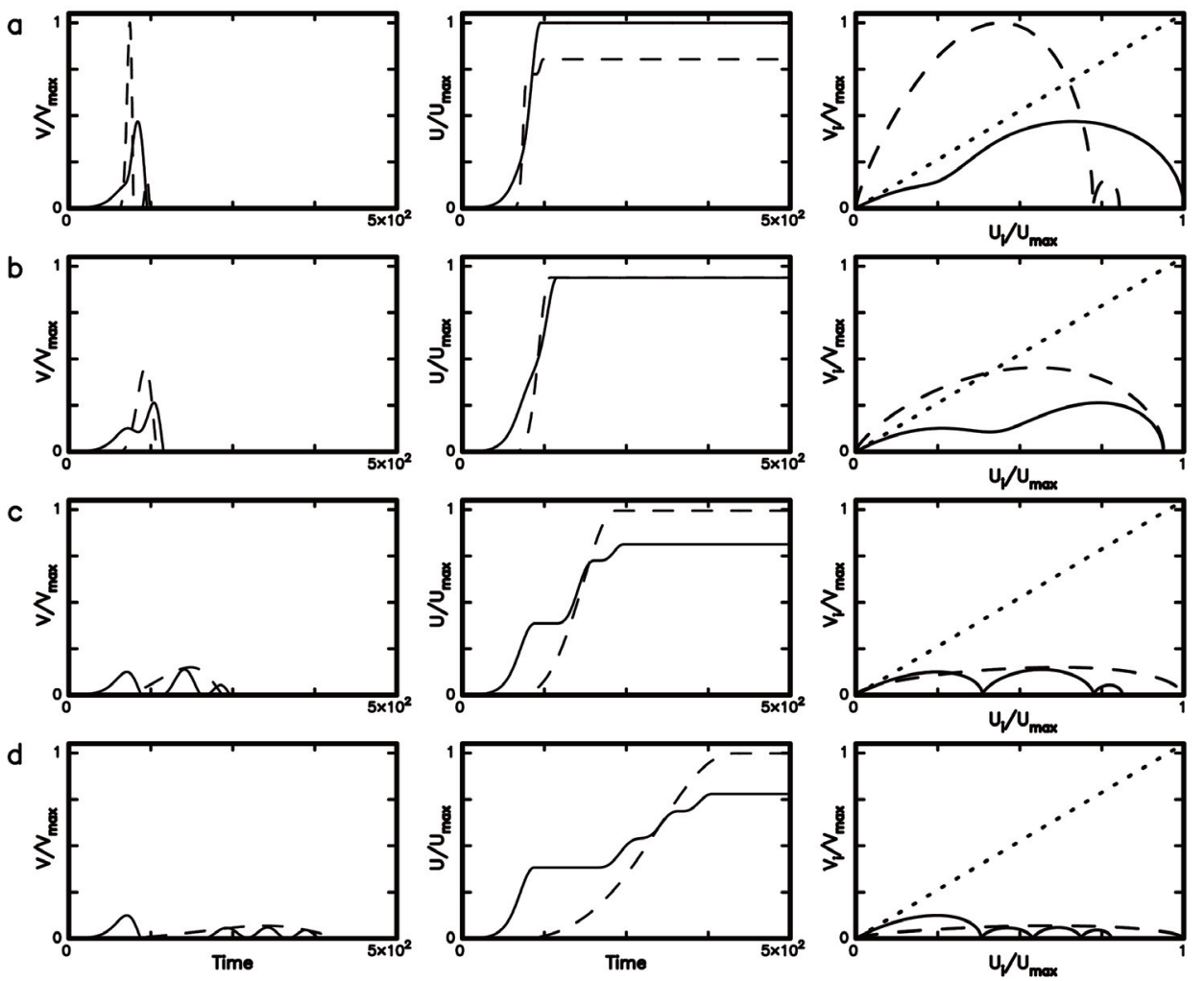

Figure 17. The time sequences of $V / V_{\max }$ and $U / U_{\max }$ and the phase portraits of $V / V_{\max }$ versus $U / U_{\max }$ of the two sliders (solid line for slider 1 and dashed line for slider 2): (a) for $\mu=0.1$, (b) for $\mu=1$, (c) for $\mu=10$, and (d) for $\mu=50$ when $s=0.48, f_{o 1}=1.00$ and $f_{o 2}=1.09$ (with $\phi=1.09$ ), $U_{c 1}=U_{c 2}=0.5$ (with $\psi=1$ ), and $\eta_{1}=\eta_{2}=0$.

Acknowledgments. I would like to express my deep thanks to the reviewer and Editor Prof. Bizzarri for significant and valuable comments, which help me to improve the manuscript. This study was financially supported by the Central Weather Bureau (Grand No.: MOTC- CWB-107-E-02), Academia Sinica, and the Ministry of Science and Technology (Grand No.: MOST-106-2116-M-001-005), Taiwan, ROC. 


\section{Inertial Effect on Interaction between Two Faults}

\section{References}

Abe, Y. and N. Kato (2013). Complex earthquake cycle simulations using a two- degree-of-freedom spring-block model with a rate- and state-friction law. Pure Appl. Geophys., 170, 745-765.

Bizzarri, A. (2009). What does control earthquake ruptures and dynamic faulting?. A review of different competing mechanism. Pure Appl. Geophys., 166, 741-776.

Brietzkel, G.B. and Y. Ben-Zion (2006). Examining tendencies of in-plane rupture to migrate to material interfaces. Geophys. J. Int. 167, 807-819, doi:10.1111/j.1365- 246X.2006.03137.x.

Brun, J.L. and A.B. Gomez (1994). A four-parameter, two degree-of-freedom block- spring model: Effect of the driver velocity. Pure Appl. Geophys., 143, 633-653.

Burridge, R. and L. Knopoff (1967). Model and theoretical seismicity. Bull. Seism. Soc. Am., 57(3), 341-371.

Byerlee, J.D. (1968). Brittle-ductile transition in rocks. J. Geophys. Res., 73, 4711-4750.

Carlson, J.M., J.S.Lange, B.E. Shaw, and C. Tang (1991). Intrinsic properties of a Burridge-Knopoff model of an earthquake fault. Phys. Rev. A, 44, 884-897.

Chen, Y.T. and L. Knopof (1986). The quasistatic extension of a shear crack in a viscoelastic medium. Geophys. J. R. astro. Soc., 87, 1025-1039.

Galvanetto, U. (2002). Some remarks on the two-block symmetric Burridge-Knopoff model. Phys. Letts. A, 293, $251-259$.

$\mathrm{Gu}$, J.C., J.R. Rice, A.L. Ruina, and S.T. Tse (1984). Slip motion and stability of a single degree of freedom elastic system with rate and state dependent friction. J. Phys. Solid, 32, 167-196.

Huang, J. and D.L. Turcotte (1990). Are earthquakes an example of deterministic chaos?. Geophys. Res. Letts., 17(3), 223-226.

Huang, J. and D.L. Turcotte (1992). Chaotic seismic faulting with a mass-spring model and velocity-weakening friction. Pure Appl. Geophys., 138(4), 549-589.

Hudson, J.A. (1980). The excitation and propagation of elastic waves. Cambridge Monographs on Mechanics and Applied Mathematics, Cambridge Univ. Press 224pp

Jaege,r J.C. and N.G.W. Cook (1977). Fundamentals of Rock Mechanics. Chapman and Hall, London, John Wiley \& Sons Inc., New York, 585pp.

Jeffreys, H. (1942). On the mechanics of faulting. Geol. Mag., 79, 291-295.

King, G.C.P. and M. Cocco (2001). Fault interaction by elastic stress changes: New clues from earthquake sequences. Adv. Geophys., 44, 1-38, doi:10.1016/S0065-2687(00)80006-0.

Kittel, C., W.D. Knight, and M.A. Ruderman (1968). Mechanics. Berkeley Physics Course, Vol. 1, McGraw-Hill Book Co., New York, NY, 480pp.

Lee, S.J., B.S. Huang, M. Ando, H.C. Chiu, and J.H. Wang (2011). Evidence of large scale repeating slip during the 2011 Tohoku-Oki earthquake. Geophys. R. Letts. 38, L01119, doi:10.1029/2011GL049584.

Lorenz, E.N. (1963). Deterministic non-periodic flow. J. Atmos. Sci., 20(2), 130-141.

Nur, A. (1978). Non-uniform friction as a physical basis for earthquake mechanics. Pure Appl. Geophys., 116, $964-991$.

Nussbaum, J. and A. Ruina (1987). A two degree-of-freedom earthquake model with static/dynamic friction. Pure Appl. Geophys., 125(4), 629-656.

Pérez Pascual, R. and J. Lomnitz-Adler (1988). Coupled relaxation oscillators and circle maps. Physica D, 30, 61-82.

Press, W.H., B.P. Flannery, S.A. Teukolsky, and W.T. Vetterling (1986). Numerical Recipes. Cambridge Univ. Press, Cambridge, 818.

Rice, J.R. (2006). Heating and weakening of faults during earthquake slip. J. Geophys. Res., 111, B05311, doi:10.1029/2005JB004006.

Savage, J.C. and A.H. Lachenbruch (2003). Consequences of viscous drag beneath a transform fault. J. Geophys. Res., 108(B1), 2025, doi:10.1029/2001JB000711.

de Sousa Vieira, M. (1999). Chaos and synchronized chaos in an earthquake model. Phys. Rev. Letts., 82(1), 201-204.

Spray, J.G. (1993). Viscosity determinations of some frictionally generated silicate melts: Implications for fault zone rheology at high strain rates. J. Geophys. Res., 98(B5), 8053-8068.

Steacy, S., J. Gomberg, and M. Cocco (2005). Introduction to special section: Stress transfer, earthquake triggering, and time-dependent seismic hazard. J. Geophys. Res., 110, B05S01, doi:10.1029/2005JB003692.

Thompson, J.M.T. and H.B. Stewart (1986). Nonlinear Dynamics and Chaos. John Wiley and Sons, New York, 376. Turcotte, D.L. (1992). Fractals and chaos in geology and geophysics. Cambridge Univ. Press, London, 221. 


\section{Jeen-Hwa Wang}

Turcotte, D.L. and G. Schubert (1982). GEODYNAMICS - Applications of Continuum Physics to Geological Problems. Wiley, 450pp.

Wang, J.C., C.F. Shieh, and J.H. Wang (2013). A study of interactions between thrust and strike-slip faults. Terr. Atmos. Ocean. Sci., 24(5), 809-825 doi:10.3319/TAO. 2013.05.08.02(T).

Wang, J.H. (1995). Effect of seismic coupling on the scaling of seismicity. Geophys. J. Int., 121, 475-488.

Wang, J.H. (2000). Instability of a two-dimensional dynamical spring-slider model of an earthquake fault. Geophys. J. Int., 143, 389-394.

Wang, J.H. (2006). Energy release and heat generation during the 1999 Chi-Chi, Taiwan, earthquake. J. Geophys. Res., 111, B11312, doi:10.1029/2005JB004018.

Wang, J.H. (2007). A dynamic study of the frictional and viscous effects on earthquake rupture: a case study of the 1999 Chi-Chi earthquake, Taiwan. Bull. Seism. Soc. Am., 97(4), 1233-1244.

Wang, J.H. (2009). Effect of thermal pressurization on the radiation efficiency. Bull. Seism. Soc. Am., 99(4), 2293-2304.

Wang, J.H. (2012). Some intrinsic properties of the two-dimensional dynamical spring-slider model of earthquake faults. Bull. Seism. Soc. Am., 102(2), 822-835

Wang, J.H. (2013). Stability analysis of slip of a one-body spring-slider model in the presence of thermal pressurization. Ann. Geophys., 56(3), R0332, doi:10. 4401/ag-5548.

Wang, J.H. (2016). Slip of a one-body dynamical spring-slider model in the presence of slip-weakening friction and viscosity. Ann. Geophys., 59(5), S0541, doi:10.4401/ag-7063.

Wang, J.H. (2017a). Multi-stable slip in a one-degree-of-freedom spring-slider model with thermal-pressurized friction and viscosity. Nonl. Processes Geophys., 24, 467-480 doi.org/10.5194/npg-24-467-2017.

Wang, J.H. (2017b). Slip of a two-degree-of-freedom spring-slider model in the presence of slip-weakening friction and viscosity. Ann. Geophys., 60(6), S0659, doi:10.4401/ag-7295.

Wells, D.L. and K.J. Coppersmith (1994). New empirical relationships among magnitude, rupture length, rupture width, rupture area, and surface displacement. Bull. Seism. Soc. Am., 84(4), 974-1002. 